\title{
Moisture absorption characteristics and mechanical degradation of composite lattice truss core sandwich panel in a hygrothermal environment
}

\author{
Jie Mei ${ }^{\mathrm{a}}$, P.J. Tan ${ }^{\mathrm{c}}$, Jiayi Liu ${ }^{\mathrm{a} * \mathrm{~b}, \mathrm{c}, \mathrm{d}}$, Zhenping He ${ }^{\mathrm{a}}$, Wei Huang ${ }^{\mathrm{a}}$

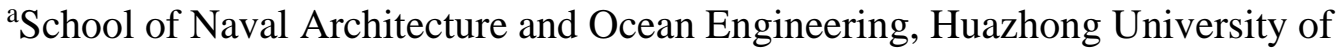 \\ Science and Technology, Wuhan 430074, PR China \\ ${ }^{\mathrm{b}}$ Collaborative Innovation Center for Advanced Ship and Deep-Sea Exploration \\ (CISSE), Shanghai 200240, PR China \\ ${ }^{\mathrm{c}}$ Department of Mechanical Engineering, University College London, Torrington \\ Place, London WC1E 7JE, UK \\ ${ }^{\mathrm{d}}$ Hubei Key Laboratory of Naval Architecture and Ocean Engineering Hydrodynamics \\ (HUST), Wuhan 430074, PR China
}

\begin{abstract}
This paper investigates the impact of hygrothermal aging upon the compressive mechanical properties of carbon fiber reinforced plastics (CFRP) composite sandwich panels with a tetrahedral truss core. Gravimetric absorption data, through accelerated moisture absorption tests, were presented for three different temperatures of $30^{\circ} \mathrm{C}, 55^{\circ} \mathrm{C}$ and $80^{\circ} \mathrm{C}$. Predictions by Fickian and Langmuir-type diffusion models were compared to evaluate which one best describe the moisture diffusion process in the sandwich panels. A subroutine implementing the Langmuir-type diffusion model in ABAQUS was developed to simulate the variations of moisture concentration. The compressive properties of composite strut and tetrahedral truss cores sandwich panel after hygrothermal aging at different temperatures were tested, respectively. The experimental results indicated that the degradation of compressive properties of composite sandwich panel was induced by the combined effect of temperature and moisture absorption. Analytical expressions were developed to predict the compressive strength and stiffness of the sandwich panel following hygrothermal aging. A good agreement was found between the predictions by finite element, analytical model and experimental results.
\end{abstract}

Keywords: Composites; Lattice materials; Hygrothermal aging; Diffusion; Mechanical properties.

\section{Introduction}

Fiber reinforced polymer composite sandwich panel is used increasingly by the aerospace and marine industries because of their light weight (low density), high specific strength and good formability [1-3]. Sandwich panels with different core topologies (such as pyramidal, tetrahedral and corrugated cores) were previously studied for various potential engineering applications [4-6], where their static and dynamic compressive mechanical properties at room temperature were widely

\footnotetext{
* Corresponding author at: School of Naval Architecture and Ocean Engineering, Huazhong University of Science and Technology, Wuhan 430074, PR China.

E-mail address: liujiayi@hust.edu.cn (Jiayi Liu).
} 
reported [7-10]. Finnegan et al. [11] manufactured composite pyramidal truss core sandwich panel by using a snap-fit method and studied its failure mechanisms under compressive loading. They found that pyramidal truss cores sandwich panel had an advantage of high specific strength. Qi et al. [12] embedded a lightweight quadrangular-prism insert into the pyramidal truss core sandwich panel to increase the reliability of the joint between the face sheets and sandwich core; this has an effect of increasing the shear strength and specific strength compared to standard honeycomb-core sandwich panel.

As polymer composites are often used in high temperature and humid environment, their hygrothermal performance is of concern to designers. The mechanism through which water molecules ingress into polymeric composites is influenced by the molecular microstructures and chemical kinetics [13]; it is also well known that excessive moisture in polymers can lead to degradation of their mechanical strength. Mansouri et al. [14] have shown that the flexural properties of mixed short fibre/woven composites are significantly affected by aging, medium (sea water or distilled water) and temperature. To analyze how the elastic properties of natural fiber reinforced composites change in a hygrothermal environment, Tian et al. [15] developed a nonlinear constitutive model that considers matrix cracking, fiber-matrix debonding and microstructural changes to the fibers. The model provides insight into the competing effects between the different mechanical properties of the natural fibers and it was applied to assess the fiber durability in a hygrothermal environment over the whole ageing process. Tual et al [16] reported the effects of hygrothermal aging on the tensile properties of unidirectional CFRP epoxy-matrix composites where they found a significant knockdown of its tensile strength; by contrast, its corresponding tensile modulus appears un-affected. On the other hand, Arhant et al. [17] found that both the compressive modulus and strength of CFRP decreases with age. One possible reason for the difference between the effects of hygrothermal aging on the tensile and compressive properties of CFRP is due to the increased elastic microbuckling of the carbon fiber because moisture induces matrix softening. Unlike metals, both temperature and moisture absorption can have a significant deleterious effect on the mechanical properties of polymer matrix composite materials [18-19], and the kinetics of moisture diffusion is consequently an important issue. A Fickian diffusion model is often used, where the moisture diffusion is assumed to be driven by the gradient of the moisture concentration [20-21]; however, Bond [22] has found that a non-fickian diffusion model is much more suitable for composites. Crace et al. [23] used a three-dimensional hindered diffusion equation to model moisture absorption in composites that exhibited Fickian and non-Fickian behavior; a non-Fickian diffusion model was found to more accurately quantify the moisture diffusion process. Wang et al. [24] developed a multi-scale model that couples the moisture diffusion to the internal stresses, caused by the moisture absorption, in laminated plates during hygrothermal expansion where the model was shown to characterize the anomalous moisture diffusion behaviors accurately compared to the Fickian diffusion model. Roy [25] et al. proposed a simple yet robust methodology to characterize non-Fickian diffusion coefficients using the weight gain data of the polymer measured from experiment.

Hitherto, the hygrothermal aging of composite lattice truss cores sandwich panel has not been studied. As lightweight sandwich panels with lattice truss cores are used increasingly in aircrafts and naval vessels that operates in extreme environment, a good understanding of their mechanical behavior to hygrothermal aging is necessary to avoid in-service catastrophic failure. In this paper, moisture absorption experiments 
were carried out on CFRP composite lattice truss cores sandwich panel at different temperatures to investigate their moisture absorption behavior, and its effect upon their compressive mechanical properties. Both the Fickian and non-Fickian diffusion models were employed to characterize the diffusion coefficients of the sandwich panel, and the results compared to the experimental data to select the most appropriate diffusion model. The compressive mechanical properties of the sandwich panel were measured following hygrothermal aging and an analytical model is developed to predict the degradation of mechanical properties under the hygrothermal environment.

\section{Experimental methods}

\subsection{Material preparation}

The tetrahedral truss cores sandwich panels were made from CFRP prepregs with epoxy resin and manufactured by a hot-press molding technique through a customized mold as shown in Fig. 1. Fig. 2 shows a schematic of the fabrication process. Composite struts, produced by rolling carbon/epoxy prepregs into a cylinder, were prepared in the form of a unidirectional cylinder with fibers aligned along its axis. The struts were placed into the semi-cylinder grooves of the mold as shown in Fig. 2a. By tightening the mold in the direction shown, the corresponding semi-cylindrical grooves in adjacent unit molds combine into uniform cylinders. The top and bottom face sheets with layers of $[0 / 90 / 0 / 90 / 0]_{\text {s }}$ were stacked on the outer surfaces of the mold after all of composite struts were positioned into the grooves. The ends of each strut were embedded into the mid-plane of face sheets as shown in Fig. 2b. The assembled molds, with preformed sandwich panel, were then cured at $125^{\circ} \mathrm{C}$ for $1.5 \mathrm{~h}$ as shown in Fig. 2c. Uniform pressures were applied to both the top and bottom face sheets during curing to assist with the hot pressing of the face sheets. The result is a tetrahedral truss cores sandwich panel (Fig. 2e) when the molds are removed as shown in Fig. 2d. A total of 30 sandwich panels were fabricated. In addition, standalone composite struts were also fabricated using the same procedure for post hygrothermal aging mechanical testing.

The composite sandwich panels were machined into rectangular specimens as shown in Fig. 3a. Each specimen consisted of 8 complete unit cells (dotted yellow box in Fig. 3a), 2 incomplete unit cells (indicated by dotted green boxes in Fig. 3a), and top and bottom face sheets. The thickness $(\delta)$ of the face sheets is $0.85 \mathrm{~mm}$. The length $\left(L_{1}\right)$ and width $\left(L_{2}\right)$ of the face sheets are $115 \mathrm{~mm}$ and $110 \mathrm{~mm}$, respectively, both are greater than the dimensions of its representative unit cell. The additional material (outside that indicated by the dotted black box in Fig. 3a) along the edges of the face sheets serves to protect the truss cores during cutting. Fig. 3b shows a schematic of a representative unit cell for the tetrahedral truss core sandwich panel. The unit cell consists of three composite struts of length $l=19.63 \mathrm{~mm}$ and diameter $d=3.00 \mathrm{~mm}$. Its relative density $\bar{\rho}$ is defined as the ratio of the volume of the truss core to that of the unit cell. The relative density $\bar{\rho}$ of the tetrahedral truss core, without the face sheets, is

$$
\bar{\rho}=\frac{3 \pi d^{2}}{8 \sqrt{3} k^{2} \sin \alpha+6 \sqrt{3} l^{2} \cos ^{2} \alpha \sin \alpha+24 k l \cos \alpha \sin \alpha}
$$

where the inclined angle $\alpha$ is $60^{\circ}$ and the distance $k$ between the ends of struts is $9 \mathrm{~mm}$. From Eq (1), the value of $\bar{\rho}$ is $2.31 \%$. The height of truss core $h_{c}$ is $17.0 \mathrm{~mm}$ and the height of composite sandwich panel $H$ is $18.7 \mathrm{~mm}$. If the face sheets are considered in the calculation of relative density, then the relative density $\bar{\rho}_{r}$ is 


$$
\bar{\rho}_{r}=\frac{n_{s t} \pi d^{2} \cdot h_{c} / 4 \sin \alpha}{L_{1} L_{2} \cdot h_{c}}=\frac{n_{s t} \pi d^{2}}{4 \sin \alpha L_{1} L_{2}}
$$

where $n_{s t}$ is the number of the struts in a specimen. The total number of struts is 26 for each specimen. It is worth highlighting that $\bar{\rho}_{r}$ is strongly affected by the dimensions of face sheet and, consequently, their variations in the cutting process.

\subsection{Accelerated moisture diffusion tests}

Natural hygrothermal aging of CFRP is a long-term process [18]. In order to accelerate the moisture diffusion process in an experimental setting, the sandwich panels were incubated in the water bath, see Fig. 4a, at three different constant temperature $T$ of $30^{\circ} \mathrm{C}, 55^{\circ} \mathrm{C}$ and $80^{\circ} \mathrm{C}$. Accelerated moisture diffusion tests were conducted according to the ASTM D5229 [26]. An electronic balance with an accuracy of $0.1 \mathrm{mg}$ (see Fig. $4 \mathrm{~b}$ ) was used to measure the mass of the specimen. The composite sandwich panels and individual composite struts were both placed into the water bath for accelerated hygrothermal aging after they were oven dried. The mechanical properties of the composite struts were tested post hygrothermal aging they will be used as inputs to the analytical model to be developed later in Section 3.2.

The front and back face of both face sheets, including the constituent struts, were uniformly exposed to water as shown in Fig. 4c. The length of the composite struts in all specimens is $18.0 \mathrm{~mm}$. As the two ends of each composite strut were embedded into the face sheets of the sandwich panel, only its exposed circumferential outer surface is available for moisture exchange. To replicate this, the two ends of every struts were sealed with silicone rubber during accelerated ageing.

The total mass of the composite sandwich panels $W$ were monitored during the moisture absorption test but not its individual composite struts since their initial mass were only $0.2 \mathrm{~g}$, which is less than the minimum value stipulated by ASTM D5229 [26]. The time interval $t_{p}$ between measurement is $48 \mathrm{~h}-$ this was obtained using $t_{P}=$ 0.04. $\delta^{2} / D$ given in ASTM D5229, where the moisture diffusion coefficient $D$ is $1.2 \times 10^{-3} \mathrm{~mm}^{2} / \mathrm{h}$ [27]. The moisture content $M_{t}$ of each specimen is calculated through

$$
M_{t}=\frac{W_{t}-W_{0}}{W_{0}} \times 100 \%
$$

where $W_{t}$ and $W_{0}$ denote the mass of wet specimen at time $t$ and oven-dried specimen, respectively. In general, moisture absorption by CFRP follows a single phase Fickian behavior in the short to medium term [20-21]; this is unlike its long-term behavior because of hydrolysis of the epoxy resin. According to the ASTM D5229 [26], effective moisture equilibrium is reached when

$$
\left|M_{t_{i}}-M_{t_{i-1}}\right|<0.020 \%
$$

where $M_{t_{i}}-M_{t_{i-1}}$ is the change in moisture content over an interval of time. An acceptable equilibrium value can be obtained according to the above criterion for a material which has an equilibrium value of at least $0.5 \%$. Note, however, that Eq. (4) is based on a flat panel and is always satisfied in our case, whereas the $M_{t}$ versus $\sqrt{t}$ curves for the sandwich specimens, plotted later in Fig. 10, will show that their moisture content increases continuously. Since the composite sandwich panel is composed of both panels and cylindrical struts, the temporal evolution of its moisture content were measured experimentally and their behaviour quantified through 
appropriate diffusion models to be developed later. The specimens were kept in the water bath until they reach an effective equilibrium moisture content or a stable diffusion rate (a constant slope of $M_{t}$ versus $\sqrt{t}$ curve). The composite sandwich panel were aged for $1470 \mathrm{~h}$ at $30^{\circ} \mathrm{C}$ and $55^{\circ} \mathrm{C}$; a shorter ageing time of $960 \mathrm{~h}$ was chosen for $80^{\circ} \mathrm{C}$.

\subsection{Mechanical testing}

Hygrothermal aging of CFRP is influenced by the combined effects of temperature and moisture content [13-18]. Uniaxial compression tests, along the thickness $(z)$ direction of each panel, were performed at various time points for each hygrothermal condition. The time points were determined by the moisture content in the panel and their changing trend. At $30^{\circ} \mathrm{C}$ and $55^{\circ} \mathrm{C}$, compression tests were performed at $510 \mathrm{~h}, 960 \mathrm{~h}$ and $1470 \mathrm{~h}$ (from when the specimens were first placed into the water bath). At $80{ }^{\circ} \mathrm{C}$, however, because of the higher moisture diffusion coefficient and ageing rate, the three time points chosen were $240 \mathrm{~h}, 510 \mathrm{~h}$ and $960 \mathrm{~h}$ instead. Three sandwich specimens were tested at each time point and temperature.

Compression testing of the individual struts and sandwich panels were conducted on a universal testing machine shown in Fig. 5. Unidirectional compression of the struts was also performed according to ASTM E2954 [28]. A customized fixture was designed to support the two ends of the composite strut, giving a gauge length $h_{s}$ of $13 \mathrm{~mm}$, see Fig. 5a. The compressive modulus $E_{\text {strut }}(t, T)$ and strength $\sigma_{\text {strut }}(t, T)$ of each composite strut was measured. Out-of-plane compression of the sandwich panels were performed in accordance to the ASTM C365 [29], see Fig. 5b. Nominal compressive stress-strain curves were obtained for the sandwich panels. For comparison purposes, the mechanical properties of a single strut and composite sandwich panel, without hygrothermal aging, were also measured. A loading rate of $0.5 \mathrm{~mm} / \mathrm{min}$ was used in all the uniaxial compression tests. A total of five single struts were tested at each time point and temperature.

\section{Analytical and numerical modelling}

\subsection{Moisture diffusion}

To investigate how moisture causes degradation of material properties (carbon fiber/epoxy composites) in the sandwich panel, the moisture absorption dynamics and equilibrium moisture content (EMC) must be known. A Fickian diffusion model is often used to model the moisture diffusion process in polymers and polymer matrix composites [21, 30-31]. However, the long-term moisture uptake of CFRP in a hygrothermal environment does not typically obey the single phase Fickian diffusion law [23, 32]. Several non-Fickian diffusion models, such as the Langmuir-type diffusion model [33], dual Fickian diffusion model [34] and exponential Fickian model [35], were previously proposed to explain the anomaly. In this study, both Fickian and non-Fickian (Langmuir-type) diffusion models will be used to fit the experimental $M_{t}$ versus $\sqrt{t}$ curves obtained for the sandwich panels; and an assessment will be made on which of the two models best describe the moisture diffusion process.

Figure 6 shows a representative unit cell for the sandwich panel where the exposed surfaces (to moisture diffusion) were marked in purple. The total moisture uptake is the sum of the moisture accumulation of the face sheets and the tetrahedral 
truss cores as shown in Fig. 6b-c. Since the panels are $115\left(L_{1}\right) \mathrm{mm} \times 110\left(L_{2}\right)$ $\mathrm{mm} \times 18.7(H) \mathrm{mm}$ in size and the thickness $\delta$ of the face sheets is $0.85 \mathrm{~mm}$, its width $L_{2}(=110 \mathrm{~mm})$ to thickness $\quad(=0.85 \mathrm{~mm})$ ratio exceeds 100 . According to ASTM D5229 [26], moisture transport across the edges of the face sheets can, therefore, be neglected and only diffusion through its thickness $(z)$ direction - direction of the fibers that were aligned along the $x$ - and $y$-axes - need to be considered. Since the two ends of each strut were embedded into the face sheets, only its circumferential surface is available for moisture absorption, i.e. through the local radial direction ( $r$-axis) as shown in Fig. 6c. Since the fibers in each strut are aligned along the local $\chi$-axis, moisture diffusion is assumed to be perpendicular to the circumferential surface of the strut. The above simplifications reduce the problem to a one-dimensional (1D) case, where only moisture diffusion perpendicular to the fiber direction is considered. In general, however, the diffusion rates of fiber reinforced polymer material are anisotropic.

If $M_{t}^{f s}$ and $M_{t}^{s t}$ is the moisture content of the top (or bottom) face sheet, and a strut at any time $t$, respectively, then, the total moisture content of the sandwich panel is

$$
M_{t}=\frac{2 \cdot m_{f s} M_{t}^{f s}+n_{s t} \cdot m_{s t} M_{t}^{s t}}{2 \cdot m_{f s}+n_{s t} \cdot m_{s t}}
$$

where $m_{f s}$ is mass of a face sheet, $m_{s t}$ is the mass of a strut and $n_{s t}$ is the total number of struts in a sandwich specimen. Let $\rho_{m}$ denotes the density of the parent material, then $m_{f s}$ and $m_{s t}$ are given by

$$
\begin{array}{r}
m_{f s}=\rho_{m} \cdot \delta \cdot L_{1} L_{2} \\
m_{s t}=\frac{\rho_{m} h_{c} \pi d^{2}}{4 \sin \alpha}
\end{array}
$$

Substituting Eqs. (6) and (7) into (5) gives

$$
M_{t}=\frac{2 \varsigma M_{t}^{f s}+\bar{\rho}_{r} M_{t}^{s t}}{2 \varsigma+\bar{\rho}_{r}}
$$

where $\varsigma=\delta / h_{c}$ is the ratio of the face sheet thickness to that of the truss cores; and $\bar{\rho}_{r}$ is relative density of the sandwich panel given in Eq. (2). To obtain the temporal variation for the total moisture content, analytical expressions must be derived for $M_{t}^{f s}$ and $M_{t}^{s t}$ (in Eq. 5) by assuming that moisture diffusion obeys a Fickian and a Langmuir-type diffusion model in the next two subsections.

\subsubsection{Fickian diffusion model}

The analytical expression for the temporal variation of the moisture content $M_{t}$ in a sandwich panel, following a Fickian diffusion process, can be obtained by substituting $M_{t}^{f s}$ and $M_{t}^{s t}$ (see Appendix A for these expressions) into Eq. (8) and, simplifying, to give

$$
M_{t}=\frac{M_{\infty}\left(2 \varsigma G_{1}(t, T)+\bar{\rho}_{r} G_{2}(t, T)\right)}{2 \varsigma+\bar{\rho}_{r}}
$$

where $G_{1}(t, T)$ and $G_{2}(t, T)$ are given, respectively, by 


$$
\begin{aligned}
& G_{1}(t, T)=1-\frac{8}{\pi^{2}} \sum_{s=1}^{\infty} \frac{\exp \left(-s^{2} \pi^{2}\left(D(T) t / \delta^{2}\right)\right)}{s^{2}} \\
& G_{2}(t, T)=1-4 \cdot \sum_{n=1}^{\infty} \frac{\exp \left(-D(T) \mu_{n}^{2} t / R^{2}\right)}{\mu_{n}^{2}}
\end{aligned} .
$$

\subsubsection{Langmuir-type diffusion model}

The Langmuir-type diffusion model is an extension of the Fickian model proposed by Carter and Kibler [33], where the anomalous moisture absorption in epoxy is assumed to consist of mobile and bound phases. The mobile moisture molecules diffuse with a concentration-independent diffusion coefficient $D_{\gamma}$, which is dependent on temperature $T$. The bound moisture molecules are unable to move freely because of chemical interaction with the polymer matrix [23]. If $n$ is the concentration of mobile molecules and $N$ the concentration of bound molecules, then the total moisture concentration $C$ in a material is

$$
C=n+N
$$

At equilibrium,

$$
\gamma n_{\infty}=\beta N_{\infty}
$$

where $n_{\infty}$ and $N_{\infty}$ are the equilibrium mobile and bound moisture concentration, respectively; $\gamma$ is the probability of a mobile water molecule becoming bound per unit time; and, $\beta$ is the probability of a bound water molecule becoming mobile per unit time.

An analytical expression for the temporal variation of the moisture content $M_{t}$ in a sandwich panel, where diffusion follows a Langmuir-type process, can be obtained by substituting expressions for $M_{t}^{f s}$ and $M_{t}^{s t}$ (see Appendix B for these expressions) into Eq. (8) to give

$$
M_{t}=\frac{M_{\infty}\left(2 \varsigma G_{3}(t, T)+\bar{\rho}_{r} G_{4}(t, T)\right)}{2 \varsigma+\bar{\rho}_{r}}
$$

where

$$
\begin{aligned}
& G_{3}(t, T)=1-\frac{8}{\pi^{2}} \sum_{\mathrm{s}=1}^{\infty} \frac{\omega_{s}^{+} e^{-\omega_{s}^{-} t}-\omega_{s}^{-} e^{-\omega_{s}^{+} t}}{s^{2}\left(\omega_{s}^{+}-\omega_{s}^{-}\right)}+\frac{8}{\pi^{2}}\left(\frac{k_{p} \beta}{\gamma+\beta}\right) \sum_{s=1}^{\infty \text { (odd) }} \frac{e^{-\omega_{s}^{-} t}-e^{-\omega_{s}^{+} t}}{\left(\omega_{s}^{+}-\omega_{s}^{-}\right)} \\
& G_{4}(t, T)=1-4 \sum_{n=1}^{\infty} \frac{\left(\zeta_{n}^{+} e^{-\zeta_{n}^{-} t}-\zeta_{n}^{-} e^{-\zeta_{n}^{+} t}\right)}{\mu_{n}^{2}\left(\zeta_{n}^{+}-\zeta_{n}^{-}\right)}+4\left(\frac{k_{c} \beta}{\gamma+\beta}\right) \sum_{n=1}^{\infty} \frac{\left(e^{-\zeta_{n}^{-} t}-e^{-\zeta_{n}^{+} t}\right)}{\left(\zeta_{n}^{+}-\zeta_{n}^{-}\right)}
\end{aligned}
$$

\subsubsection{Temperature-dependent diffusion parameters}

Recall that we wish to assess how well the experimental data for the moisture content $M_{t}$ versus $\sqrt{t}$ fits the prediction by Eqs. (9) or (14). Since the equilibrium moisture content $M_{\infty}$, diffusion coefficient of Fickian diffusion $D$, diffusion coefficient of Langmuir-type diffusion $D_{\gamma}$ and Langmuir-type diffusion parameters $\gamma$ and $\beta$ are all temperature-dependent parameters, it is necessary to fit the experimental data by an empirical Arrhenius-type law. 
The empirical Arrhenius-type law for diffusion parameters $\Gamma=M_{\infty}, D, D_{\gamma}, \gamma$ or $\beta$ which depend on temperature $T$ is given by [36]

which can also be written as

$$
\Gamma=\Gamma_{0} \exp \left(-\frac{E_{\Gamma}}{R_{c} T}\right)
$$

$$
\ln (\Gamma)=\ln \left(\Gamma_{0}\right)-\frac{E_{\Gamma}}{R_{c} T}
$$

where $\Gamma_{0}$ is the corresponding diffusion parameter when $T \rightarrow \infty ; R_{c}$ is the universal gas constant $\left(R_{c}=8.32 \times 10^{-3} \mathrm{~kJ} / \mathrm{mol}\right)$; and $E_{\Gamma}$ is the equilibrium activation energy of the corresponding diffusion parameter.

\subsection{Compressive properties of sandwich panel}

Figure 7 shows a schematic of the deformation by a unit cell under compressive loading. The compressive force $F_{N}$ applied to the unit cell is resisted by three struts. The internal forces $N, M$ and $Q$ are the axial compressive force, bending moment and shearing force at the end of each strut, respectively. Based on small deformation theory - since this is within the elastic range - the internal forces at the end of each strut are

$$
\left\{\begin{array}{l}
N=\frac{\pi d^{2} \delta \sin \alpha}{4 l} E_{\text {strut }}(T, t) \\
M=\frac{6 I \delta \cos ^{2} \alpha}{l^{2} \sin \alpha} E_{\text {strut }}(T, t) \\
Q=\frac{12 I \delta \cos \alpha}{l^{3}} E_{\text {strut }}(T, t)
\end{array}\right.
$$

where $d$ and $I$ are the diameter and the moment of inertia, respectively; $\delta$ is the displacement of the unit cell along the thickness direction; $E_{\text {strut }}(T, t)$ is the compressive modulus of the strut expressed as a function of immersion time $t$ and temperature $T$. Through force equilibrium, the compressive force acting on the unit cell is

$$
F_{N}=3(N \sin \alpha+Q \cos \alpha)
$$

From the constitutive relation, the equivalent compressive stiffness of a unit cell can be, therefore, be written as

$$
E_{z}(T, t)=\frac{F_{N} h}{a b \cdot \delta}=\frac{3(N \sin \alpha+Q \cos \alpha) h}{\delta \cdot a b}
$$

Substituting for $N$ and $Q$ using Eq. (19), one obtains

$$
E_{z}(T, t)=\bar{\rho} E_{\text {strut }}(T, t) \sin ^{4} \alpha \cdot(1+\Psi)
$$

where the $\bar{\rho}$ is the relative density of the tetrahedral truss core unit cell; $E_{\text {strut }}$ is the compressive modulus of composite strut; the coefficient $\Psi$ reflects the effect degree of the bending deformation in the strut on the compressive stiffness of the sandwich panel given by $\Psi=12 \cot ^{2} \alpha / \lambda^{2}$. It is worth highlighting that the slenderness ratio of a strut $\lambda=4 l / d$ has a significant effect on the value of the coefficient $\Psi$. Since our composite strut has a slenderness ratio $\lambda$ of 26.16 , leading to a coefficient $\Psi$ of 0.00584 , it can be concluded that its bending deformation is negligibly small. Since 
the tetrahedral truss core is a stretch-dominated lattice, Eq. (22) can be simplified to give the following

$$
E_{z}(T, t)=\bar{\rho} E_{\text {strut }}(T, t) \sin ^{4} \alpha
$$

The mode of failure by the sandwich panel under compression is node failure caused by axial compressive stresses acting at both ends of the composite strut given by

$$
\sigma_{\chi}=N / \pi(d / 2)^{2}
$$

When the axial compressive stress $\sigma_{\chi}$ reaches the compressive strength of the strut $\sigma_{\text {strut }}(T, t)$, the sandwich panel is deemed to have failed. Substituting $N$ from Eq. (19) into Eq. (24) gives the compressive strength $\sigma_{z}(T, t)$ of the sandwich panel as

$$
\sigma_{z}(T, t)=\bar{\rho} \sigma_{\text {strut }}(T, t) \sin ^{2} \alpha
$$

\subsection{Predictive model for ageing}

The hygrothermal aging of CFRP is affected by numerous factors such as chemical reactions in the matrix resin [32], progression of micro-cracks [37], debonding at the fiber/matrix interface, etc.; its resulting mechanical degradation is a complex multi-scale and multi-factor process. It was previously reported by others $[27$, 38-39] that there is a correlation between the deterioration of material properties and the mass variations in CFRP. Here, as a simplification, the compressive modulus $E_{c}(T, t)$ and strength $\sigma_{c}(T, t)$ of CFRP is considered to be dependent only on its moisture concentration $C(t, T)$ given by

$$
\begin{aligned}
& E_{c}(t, T)=f_{1}(C(t, T)) \\
& \sigma_{c}(t, T)=f_{2}(C(t, T))
\end{aligned}
$$

where $f_{1}$ and $f_{2}$ are monotonic decreasing - either a linear or exponential functions. To predict the degradation of the mechanical properties of a sandwich panel with age, the temporal variations of the strut modulus $E_{\text {strut }}(T, t)$ and strength $\sigma_{\text {strut }}(T, t)$ are needed. Since moisture concentration is non-uniform within the strut during diffusion, the degradation of its mechanical properties must also be non-uniform. Hence, the aged mechanical properties at any point of the strut over time is a function of radial $r$ and time $t$ given by

$$
\begin{aligned}
& E_{c}(r, t, T)=f_{1}(C(r, t, T)) \\
& \sigma_{c}(r, t, T)=f_{2}(C(r, t, T))
\end{aligned}
$$

where $C(r, t, T)$ is the moisture concentration given by Eq. (A-7) for Fickian diffusion and by Eqs. (A-26-A-27) for Langmuir-type diffusion. By integrating the uniaxial compressive stress $\sigma_{\chi}(r, t, T)=E_{c}(r, t, T) \varepsilon_{\chi}$ at the time when the strut fails, its strength $\sigma_{\text {strut }}(T, t)$ can be expressed as

$$
\sigma_{\text {strut }}=\frac{\int_{0}^{2 \pi} \int_{0}^{R} f_{1}(C(r, t, T)) \varepsilon_{\chi} \cdot r d r d \varphi}{\pi R^{2}}
$$

where $\varepsilon_{\chi}$ is the uniaxial compressive strain at the peak stress. In the elastic range, a simplified model is used to determine $\sigma_{\chi}$ and $\varepsilon_{\chi}$ by assuming that the maximum 
strain $\varepsilon_{\chi}$ are identical along the radial direction of the strut. Hence, damage initiates at a site where the stress $\sigma_{\chi}$ is first equal to the compressive strength $\sigma_{c}$ and $\varepsilon_{\chi}$ is given by

$$
\varepsilon_{\chi}=\frac{\sigma_{c}\left(r_{s}, t, T\right)}{E_{c}\left(r_{s}, t, T\right)}=\frac{f_{2}\left(C\left(r_{s}, t, T\right)\right)}{f_{1}\left(C\left(r_{s}, t, T\right)\right)}
$$

where $r_{s}$ is the coordinate when damage first initiates. Note that $r_{s}$ is different for different moisture distribution. The modulus $E_{\text {strut }}(T, t)$ is given by

$$
E_{\text {strut }}(T, t)=\sigma_{\text {strut }}(T, t) / \varepsilon_{\chi}
$$

Substituting Eqs. (30) and (31) into (32), one obtains the compressive modulus as follows:

$$
E_{\text {strut }}=\frac{\int_{0}^{2 \pi} \int_{0}^{R} f_{1}(C(r, t, T)) \cdot r d r d \varphi}{\pi R^{2}} .
$$

The form of $f_{1}$ can be determined by using the modulus $E_{\text {strut }}$ measured in experiment with Eq. (33). Before obtaining $f_{2}$ in Eq. (31), the coordinate $r_{s}$ needs to be determined. Typically, a numerical method is needed to evaluate $r_{s}$. In our case, however, the problem can be simplified because the distribution of moisture concentration $C(r, t)$ varies monotonically along the radial direction $r$ as shown in Fig. 8a. The variations of the degraded modulus $E_{c}$ and strength $\sigma_{c}$ along the radial direction are, also consequently monotonic through transformations given in Eq. (28-29). Hence, the stress distribution $\sigma_{\chi}(r, t)$ along the radial direction has a similar profile to that of $E_{c}$ and $\sigma_{c}$. The damage initiation site is either on the outer surface or the center of the strut as shown in Fig. 8b. The region of failure region depends on ratio of the slopes of $\sigma_{c}$ and $\sigma_{\chi}$. Failure would occur at the center of the strut if the following relationship is satisfied:

$$
\left|\frac{\mathrm{d} \sigma_{c}}{\mathrm{~d} r}\right|<\left|\frac{\mathrm{d} \sigma_{\chi}}{\mathrm{d} r}\right| .
$$

On the other hand, failure occurs at the strut surface if

$$
\left|\frac{\mathrm{d} \sigma_{c}}{\mathrm{~d} r}\right|>\left|\frac{\mathrm{d} \sigma_{\chi}}{\mathrm{d} r}\right| .
$$

\subsection{Finite element modelling}

In order to investigate the variation of moisture distribution in tetrahedral truss cores sandwich panel during the moisture diffusion, the finite elements method (FEM) is adopted to simulate the moisture diffusion process. When adopting Fickian diffusion model, the transient moisture diffusion process in the composite sandwich panel was simulated by using the ABAQUS mass diffusion analysis. Without including the combined effect of temperature and pressure, the governing equation of ABAQUS mass diffusion analysis is an extension of Fick's equations and are given as [40]

$$
\int_{V}\left(\frac{d C}{d t}+\frac{\partial}{\partial x} \cdot \mathbf{J}\right) d V=0
$$


where

$$
\mathbf{J}=-S_{b} D \cdot \frac{\partial \phi}{\partial x}
$$

where $D$ is the diffusion coefficient, $\phi=C / s_{b}$ is defined as the normalized concentration in which $C$ is the mass concentration of the diffusion material and $s_{b}$ is the solubility of it in the base material. The solubility $s_{b}$ of the moisture in the base material is supposed to be 1 . Hence, the moisture concentration on the boundary of the model is set as the equilibrium moisture concentration $C_{\infty}$ to make the maximum moisture concentration in the model less than $C_{\infty}$.

Unfortunately, Langmuir-type diffusion model was not contained in ABAQUS code. To implement the analytical equation of Langmuir-type diffusion model which is an extension of Fickian diffusion model, a user subroutine HETVAL of ABAQUS was used and a Python code was adopted for the post processing. The details of implementing Langmuir-type diffusion model are given in Appendix C.

The FEM model of composite sandwich panel is built with the dimensions of the tested specimens as shown in Fig. 9. The height of the tetrahedral truss cores is $17 \mathrm{~mm}$ and the thickness of the face sheets is $0.85 \mathrm{~mm}$ with stacking sequence of $[0 / 90 / 0 / 90 / 0]_{s}$. The length and width of the face sheets varies from $110 \mathrm{~mm}$ to $118 \mathrm{~mm}$ according to the actual dimensions of specimens in order to ensure the $\bar{\rho}_{r}$ of FEM model equating with $\bar{\rho}_{r}$ of the simulated specimen. The material properties of diffusion are considered to be orthotropic in this model. Nevertheless, the diffusion coefficient along the longitude direction of fiber is supposed to be different from that perpendicular to longitude direction. The moisture in the FEM model mostly diffused perpendicular to longitude direction of fibers. Thus, the errors induced by this simplification are negligible. The diffusion coefficient $D$ (for Fickian diffusion model) or $D_{\gamma}$ (for Langmuir-type diffusion model) used in the FEM are the measured data from the accelerate diffusion experiments. The material orientation of the face sheets and struts are shown in Fig. 9a-b, respectively. The boundary conditions of the FEM model are in accordance with these of the analytical model as shown in Fig. 6. For Fickian diffusion model, the moisture concentration on the boundary is the equilibrium moisture concentration $C_{\infty}$ which is obtained by moisture absorption experiments. But for Langmuir-type diffusion model, the moisture concentration on the boundary is the equilibrium moisture concentration of mobile water molecules $n_{\infty}$ which can be obtained by Eq. (12-13) and the measured $C_{\infty}$. For both two diffusion models, the element type of the FEM model was DC3D20 which is a 20-node quadratic heat transfer brick.

\section{Results}

\subsection{Moisture diffusion}

After accelerated moisture diffusion tests, the moisture diffusion curves $\left(M_{t}\right.$ versus $\sqrt{t}$ curves) of composite sandwich panels at three different temperatures are presented in Fig. 10. Three series of specimens are presented at each temperature $T$, named by S-1-T, S-2-T and S-3-T. In the initial part of all moisture diffusion curves, the moisture contents increase linearly, and then gradually slow down. It can be found that there is a sustained growth region of the tested moisture content at the last period 
where the slopes of the curves are stable. Least-squares regression method is applied to recover the moisture diffusion parameters of the Fickian diffusion model and the Langmuir-type diffusion model from the experimental data as shown in Fig. 11, respectively. The regression equations based on Fickian model and Langmuir-type model are respectively given in Eq. (9) and Eq. (14). The coefficient $\varsigma$ is 0.1 for all the specimens. The actual relatively densities $\bar{\rho}_{r}$ of tetrahedral truss cores are given in Table 1. The recovered moisture diffusion parameters $D(T)$ and $M_{\infty}(T)$ of Fickian diffusion model are listed Table 2. The recovered moisture diffusion parameters $D_{\gamma}(T), M_{\infty}(T), \gamma(T)$ and $\beta(T)$ of Langmuir-type diffusion model are given in Table 3. The typical fit curves of S-1 at different temperatures are given in Fig. 11. The residual error (Er) of the fit curves is defined as

$$
\mathrm{Er}=\sum_{i=1}^{n}\left(\bar{M}_{t}\left(t_{i}\right)-M_{t_{i}}\right)^{2}
$$

where $M_{t_{i}}$ is the measured moisture content at time $t_{i}$ and $\bar{M}_{t}\left(t_{i}\right)$ is the moisture content of the fit function at time $t_{i}$. Thus, for a same specimen, as the residue error gets smaller, the fitting effect gets better. The results indicate that Langmuir-type diffusion model gives better fitting in comparison with Fickian diffusion model. The fitting curves of Fick diffusion and Langmuir-type diffusion are coincident approximately with measured results in the initial stage as shown in Fig. 11. However, the model of Fick diffusion can not appropriately describe the sustained ascending of the experimental moisture contents at the last period.

The diffusion parameters are supposed to be temperature dependent following the empirical Arrhenius-type law as given in Eq. (18). With the average values of the experimental data in Table 2 and Table 3, the linear fitting functions of the diffusion parameters $\ln (D), \ln \left(D_{\gamma}\right), \ln \left(M_{\infty}\right), \ln (\gamma)$ and $\ln (\beta)$ versus the inverse temperature $1 / T$ are given in Fig. 12, respectively. Acceptable correlation coefficients (Adj. $\mathrm{R}^{2}$ ) are obtained from the linear regression analysis of the plot Arrhenius equations.

The FEM results of moisture diffusion in the sandwich panel are obtained as shown in Fig.13-14, by using the recovered diffusion parameters listed in Table 2 and Table 3. The typical moisture diffusion curve of Fickian diffusion are given in Fig. 13a, which are in good agreement with the analytical model, however, similar deviations of FEM results are found compared to the test data. From Fig.14a, The numerical moisture diffusion curve of Langmuir-type model are in good agreement with both analytical results and test data. Ignoring anomalous moisture absorption is the reason for the deviations of Fickian diffusion model. From the moisture concentration contours as shown in Fig. 13c-e, the moisture diffuse rapidly along concentration gradient until reaching the equilibrium concentration. The initial phase fits the test data well because the moisture diffusion driven by the concentration gradient is the main process. But the anomalous moisture absorption plays a major role in the last phase, which makes a sustained growth of moisture content. As shown in Fig. 14c-e for Langmuir-type diffusion, the moisture concentration reaches an approximate equilibrium quickly, however, the moisture keeps growing due to the 'sink' of the mobile moisture molecules. It can be seen that Langmuir-type diffusion model can simulate the anomalous moisture absorption caused by the interaction between the moisture and polymer matrix and is in better fit with experimental results compared with Fickian diffusion model. 


\subsection{Modulus and strength of composite strut}

The compressive properties of single composite strut were tested post the accelerated hygrothermal aging to predict the degradation of the mechanical properties. The typical stress-strain curves of single struts without aging and post aging are given in Fig. 15a. Under uniaxial compression, the main failure mode of composite strut is delamination and fracture along the longitude direction of fiber for all specimens as shown in Fig. 15b. For most specimens, the failure sites are close to the strut fixture as shown in right of Fig. 15b. After the initial local fracture of fibers appearing at the struts, the delamination along fiber direction expands rapidly.

The measured compressive strength $\sigma_{\text {strut }}(T, t)$ and compressive modulus $E_{\text {strut }}(T, t)$ of composite struts at different temperatures $T$ and immersion time $t$ are different as shown in Fig. 16. The results indicate that the hygrothermal aging at high temperature has a significant effect on the compressive properties of composite strut. There are slight reduction in the compressive strength and modulus of composite strut at $30^{\circ} \mathrm{C}$. The compressive strength and modulus had a decline in the proportion of $2.7 \%$ and $5.4 \%$ after $1470 \mathrm{~h}$, respectively. Under $55^{\circ} \mathrm{C}$ hygrothermal environment, the strength and modulus of composite strut gradually decreased as immersion time increased as well. After $1470 \mathrm{~h}$ hygrothermal condition at $55^{\circ} \mathrm{C}$, the compressive strength and modulus dropped to $88.6 \%$ and $90.3 \%$ of their original values, respectively. The average compressive strength of composite strut decreased by $26.8 \%$ and its modulus was reduced by $19.4 \%$ from $0 \mathrm{~h}$ to $960 \mathrm{~h}$ at $80^{\circ} \mathrm{C}$.

\subsection{Compression of sandwich panels}

The out-of-plane compressive stress-strain curves and typical failure modes of tetrahedral truss cores sandwich panel were collected through the experiments. As shown in Fig. 17, the typical failure mode of composite sandwich panel is node failure for all specimens. As the ends of the struts are directly embedded in the middle of face sheets, there is no strengthening structure for the nodes. Hence, the nodes between face sheets and the truss cores are the weakest regions under the out-of-plane compression. The experimental compressive stiffness and strength of composite sandwich panel are given in Table 4 with standard deviations of each group. The average values of each group are based on results of 3 specimens. All typical stress-strain curves exhibit the same trend at $30^{\circ} \mathrm{C}$ with different immersion time as shown in Fig. 18a. The stress peak of the stress-strain curves are taken as the compressive strength while the slopes of the line segment of the curves are obtained to be the stiffness of the sandwich panel. The effect of hygrothermal aging at $30^{\circ} \mathrm{C}$ on the compressive properties of the sandwich panel over immersion time are shown in Fig. 18b. It can be seen that there was no obvious mechanical degradation after $510 \mathrm{~h}$ under $30^{\circ} \mathrm{C}$ hygrothermal condition. Until $960 \mathrm{~h}$ at $30^{\circ} \mathrm{C}$, the average compressive strength and stiffness both decreased by about $2.0 \%$. When the sandwich panels were aged $1470 \mathrm{~h}$ at $30^{\circ} \mathrm{C}$, the average strength reduced by $2.7 \%$ and the average modulus reduced by $3.4 \%$, respectively. Under $50^{\circ} \mathrm{C}$ hygrothermal environment, the typical stress-strain curves are shown in Fig. 18c. As shown in Fig. 18d, the average strength decreased by $2.4 \%, 4.1 \%$ and $7.7 \%$ for the sandwich panel aged $510 \mathrm{~h}, 960 \mathrm{~h}$ and $1470 \mathrm{~h}$, respectively. Moreover, the average stiffness dropped by $5.0 \%, 6.5 \%$ and $9.6 \%$ after $510 \mathrm{~h}, 960 \mathrm{~h}$ and $1470 \mathrm{~h}$, respectively. The results of compressive properties of 
sandwich panels at $80^{\circ} \mathrm{C}$ are given in Fig. 18e-f. The average strength decreased by $9.5 \%, 17.9 \%$ and $22.1 \%$ for the sandwich panel aged $240 \mathrm{~h}, 510 \mathrm{~h}$ and $960 \mathrm{~h}$, respectively. The average compressive stiffness reduced by $10.3 \%, 16.2 \%$ and $19.0 \%$ after $240 \mathrm{~h}, 510 \mathrm{~h}$ and $960 \mathrm{~h}$, respectively. The hygrothermal aging of mechanical properties of the sandwich panel at $80^{\circ} \mathrm{C}$ was obviously more serious than that $30^{\circ} \mathrm{C}$ and $55^{\circ} \mathrm{C}$. With the measured modulus and strength of the single struts, the analytical results of the sandwich panel are obtained by Eq. (23) and Eq. (25) and compared with experimental results as shown in Fig. 18. The relative errors are acceptable for the analytical stiffness. For the fact that the stress concentration at the end of composite strut has a significant effect on the compressive strength of the composite sandwich panel, the analytical strength is slightly overestimated. However, the analytical retention ratio of compressive stiffness and strength exhibit the same variation trend with the experimental results.

\subsection{Prediction and discuss}

In this section, the undetermined parameters of the predicting method in section 3.3 are obtained by the experimental results and used to provide prediction mechanical properties of the sandwich panel. The aim of this work is to give prediction mechanical properties of the sandwich panel at any time $t$ and temperature $T$.

By accelerated moisture diffusion tests of the sandwich panels, one can obtain their moisture diffusion curves ( $M_{t}$ versus $\sqrt{t}$ curves) and temperature-dependent diffusion parameters of Fickian diffusion model and Langmuir-type diffusion model. According to the analytical results and FEM results, Langmuir-type diffusion model gives better fitting in comparison with Fickian diffusion model. According to the researches of Zafar et al. [41], the water molecules in epoxy matrix can reduce the crosslinks and the segments rigidity which could lead to decreasing of compressive properties of composite materials. Furthermore, the swelling of the matrix can induce cracks in the materials and decrease the bonding strength of the interface between fiber and matrix [42]. Thus, there is a strong connection between moisture concentration and the degradation degree of mechanical properties. Hence, with Fickian diffusion model, unreasonable results of anomalous diffusion were obtained because of ignoring the reaction between moisture and polymer matrix. Consequently, Langmuir-type diffusion model with the temperature-dependent diffusion parameters is used to characterize the diffusion process of CFRP sandwich panel.

In [38], a linear correlation between the moisture concentration and elastic properties of the composite material was verified to be useful to predict the mechanical properties aging. According to our experimental data, the linear correlation is also applicable and useful. Linear functions are adopted for $f_{1}$ and $f_{2}$ in Eq. (64-65) and written as

$$
\begin{aligned}
& E_{c}(r, t, T)=f_{1}(C(r, t, T))=u C(r, t, T)+w \\
& \sigma_{c}(r, t, T)=f_{2}(C(r, t, T))=u^{\prime} C(r, t, T)+w^{\prime}
\end{aligned}
$$

where the constants $u, u^{\prime}<0$ and $w, w^{\prime}>0$. By substituting Eq. (39) into Eq. (33), one can obtain the compressive modulus of the strut

$$
E_{\text {strut }}(t, T)=u M_{t}^{s t}+w
$$

where $M_{t}^{s t}$ is the moisture content of the strut given in Eq. (A-29) as a function of temperature $T$ and immersion time $t$. From Eq. (30-31), the compressive strength of 
the strut is given as

$$
\sigma_{\text {strut }}(t, T)=\frac{u^{\prime} C\left(r_{s}, t, T\right)+w^{\prime}}{u C\left(r_{s}, t, T\right)+w} E_{\text {strut }}(t, T)
$$

where $r_{s}$ can be 0 when Eq. (34) is satisfied or $R$ as Eq. (35) being satisfied. From the linear functions $f_{1}$ and $f_{2}$, Eq. (34-35) are respectively simplified to be

$$
\left|\frac{w}{u}\right|<\left|\frac{w^{\prime}}{u^{\prime}}\right|
$$

and

$$
\left|\frac{w}{u}\right|>\left|\frac{w^{\prime}}{u^{\prime}}\right|
$$

As the $u, w, u^{\prime}$ and $w^{\prime}$ are constants, the failure site is supposed to be identical at different time and temperature. The constants $u$ and $w$ in Eq. (41) are recovered by using the experimental modulus of the single struts given in Fig. 16a. The moisture content $M_{t}^{\text {st }}$ at different time $t$ and temperature $T$ in Eq. (41) is obtained by Eq. (A-29) with the average diffusion parameters given in Table 3. The linear fit of experimental modulus $E_{\text {strut }}$ and moisture content $M_{t}^{s t}$ of the strut is shown in Fig. 19a. To recover the constants $u^{\prime}$ and $w^{\prime}$, Eq. (41) is transformed to be

$$
\tilde{\sigma}_{\text {strut }}(t, T)=u^{\prime} C\left(r_{s}, t, T\right)+w^{\prime}
$$

where $\tilde{\sigma}_{\text {strut }}(t, T)$ is the stress at the failure site and is given as

$$
\tilde{\sigma}_{\text {strut }}(t, T)=\frac{E_{c}\left(r_{s}, t, T\right)}{E_{\text {strut }}(t, T)} \cdot \sigma_{\text {strut }}(t, T)
$$

The recovered constants $u^{\prime}$ and $w^{\prime}$ are shown in Fig. 19b. The recovered constants satisfied Eq. (86), which indicated that the initial failure site is the surface of the strut. From Fig. 19, the linear correlations given in Eq. (39-40) are proved to be reasonable.

To predict the compressive stiffness $E_{z}(t, T)$ and strength $\sigma_{z}(t, T)$ of the sandwich panel at different time $t$ and temperature $T$, the flowchart is shown in Fig. 20. First, with input immersion time $t$ and temperature $T$, the temperature-dependent diffusion parameters $M_{\infty}(T), D_{\gamma}(T), \quad \gamma(T)$ and $\beta(T)$ are obtained by Arrhenius equations as given in Eq. (18) which are the fitting results of the accelerated moisture diffusion tests. Then, the distributions of moisture concentration $C(r, t, T)$ in the strut are calculated by FEM as given in section 3.4 (or analytical solutions of Langmuir-type diffusion model as given in Eq. (A-29)). By substituting moisture concentration $C(r, t, T)$ into the recovered empirical functions Eq. (41-42), one can obtain the modulus $E_{\text {strut }}(t, T)$ and strength $\sigma_{\text {strut }}(t, T)$ of the single strut. Finally, the stiffness $E_{z}(t, T)$ and strength $\sigma_{z}(t, T)$ of the sandwich panel are gained by Eq. (23) and Eq. (25), respectively. The prediction retention ratio of stiffness $E_{z}(t, T) / E_{z}(t=0)$ and strength $\sigma_{z}(t, T) / \sigma_{z}(t=0)$ of the sandwich panel at different temperature and time are shown in Fig. 21.

It can be seen in Fig. 21a, the prediction retention ratio of stiffness are in good agreement with experimental results. However, the prediction retention ratio of strength is lower than measured results as shown in Fig. 21b. These deviations are mainly due to that we consider the failure of the sandwich panel occur at the time the 
initial damage appearing at the strut. However, the actual failure of the sandwich panel is a progressive damage process. Hence, the sandwich panel would not lose all the carrying capacity when initial damage occur at the strut. To further investigate the progressive damage process and the effect of stress concentration at the node of the sandwich panel under compression load, it is necessary to use FEM to simulate the mechanical response post hygrothermal aging.

\section{Conclusions}

In this paper, accelerated moisture diffusion tests of CFRP tetrahedral truss cores sandwich panel were conducted to investigate the moisture absorption behaviors and hygrothermal aging of mechanical properties. A method by using Fickian diffusion model and Langmuir-type moisture diffusion model were both developed to characterize the moisture diffusion parameters of the sandwich panel. The obtained moisture diffusion parameters: equilibrium moisture content $M_{\infty}$, diffusion coefficient $D$ or $D_{\gamma}$ and Langmuir parameters $\gamma$ and $\beta$ were used to conduct FEM model of moisture diffusion process. The analytical results and FEM results indicated that Langmuir-type diffusion model was more reasonable and had better fitting with the experimental results. According to the experimental results, temperature had a significant influence on the moisture absorbing behavior. The equilibrium moisture content $M_{\infty}$, diffusion coefficient $D_{\gamma}$, molecular binding probability $\gamma$ and molecular unbinding probability $\beta$ which govern absorption kinetics increased as temperature increasing and followed the Arrhenius equations.

The compressive properties of the single struts and the sandwich panel were both tested post hygrothermal aging. Multi-group of specimens at three different temperatures and different time points were conducted to investigate the effect of temperature and moisture content on the degradation of the mechanical performance. The results indicated that the hygrothermal aging of the sandwich panel had no influence on the compressive failure mode which was node failure. However, aging time and temperature had a significant effect on the reduction of mechanical properties of composite sandwich panel. The longer aging time and higher temperature resulted in higher degradation proportion of the mechanical properties. The compressive properties of composite sandwich panel decreased by about $3 \%$ at $30^{\circ} \mathrm{C}$ after $1470 \mathrm{~h}$, whereas $22.1 \%$ reduction of compressive strength was found at $80{ }^{\circ} \mathrm{C}$ after $960 \mathrm{~h}$. By considering the linear correlation between the moisture concentration and elastic properties of the composite material, a prediction method was developed to forecast the reduction of mechanical properties of composite sandwich panel. The prediction results were reasonable and useful compared with experimental results.

\section{Appendix A. Fickian diffusion model}

In principal material coordinates of face sheets, see Fig. 6b, the one-dimensional Fickian diffusion model can be written as [21]

$$
\frac{\partial C(z, t)}{\partial t}=D(T) \frac{\partial^{2} C(z, t)}{\partial z^{2}}
$$

where $C(z, t)$ is the moisture concentration at time $t$, and $D(T)$ is the diffusion coefficient which is greatly depended on the temperature $T$. For both the top and 
bottom face sheets (Fig. 6a), it shall be assumed that they are initially dry. Therefore, the boundary conditions and initial conditions are as follows:

$$
\left\{\begin{array}{l}
C(0, t)=C(\delta, t)=C_{\infty} \quad t \geq 0 \\
C(z, 0)=0 \quad 0<z<\delta
\end{array}\right.
$$

where $C_{\infty}$ is the saturated moisture concentration at temperature $T$. In practice, $C_{\infty}$ can be considered to be the equilibrium moisture content (or EMC). The solution to Eq. (A-1) can be expressed in the form of a trigonometrical series as follows [20]:

$$
C(z, t)=C_{\infty}\left(1-\frac{4}{\pi} \sum_{n=0}^{\infty} \frac{(-1)^{n}}{2 n+1} \exp \left(\frac{-(2 n+1)^{2} \pi^{2} D(T) t}{\delta^{2}}\right) \cos \frac{(2 n+1) \pi z}{2 \delta}\right)
$$

The moisture content $M_{t}^{f_{s}}$ in one of the face sheets at time $t$ is obtained by integrating $C(z, t)$ in Eq. (A-3) over the domain to give

$$
M_{t}^{f_{s}}=\frac{\int_{0}^{\delta} C(z, t) d z}{\delta}=M_{\infty}\left(1-\frac{8}{\pi^{2}} \sum_{n=0}^{\infty} \frac{\exp \left(-(2 n+1)^{2} \pi^{2}\left(D(T) t / \delta^{2}\right)\right)}{(2 n+1)^{2}}\right)
$$

where $M_{\infty}$ is the saturated moisture content of the specimen at temperature $T$.

For diffusion through struts, consider a perfect cylinder where moisture diffusion occurs in a local radial direction as shown in Fig. 6c. Rewriting Eq. (A-1) in the cylindrical coordinate system, see Fig. 6c, the Fickian diffusion equation is

$$
\frac{\partial C(r, t)}{\partial t}=D(T)\left(\frac{\partial^{2} C(r, t)}{\partial r^{2}}+\frac{1}{r} \frac{\partial C(r, t)}{\partial r}\right) .
$$

The boundary and initial conditions are

$$
\left\{\begin{array}{l}
C(R, t)=C_{\infty} ; C(0, t)<\infty \quad t \geq 0 \\
C(r, 0)=0 \quad 0 \leq r<R
\end{array}\right.
$$

where $R$ is the radius of the cylinder and $C_{\infty}$ is the saturated moisture concentration at temperature $T$. Using the method of separation of variables [20], the solution to Eq. $(\mathrm{A}-5)$ is

$$
C(r, t)=C_{\infty}\left(1-2 \sum_{n=1}^{\infty} \frac{J_{0}\left(\mu_{n} r / R\right)}{J_{1}\left(\mu_{n}\right) \cdot \mu_{n}} \exp \left(\frac{-D(T) \mu_{n}^{2} t}{R^{2}}\right)\right)
$$

where $J_{0}$ and $J_{1}$ are the Bessel function of the first kind of order zero and order one, respectively. Note that $\mu_{n}$ are the roots of the equation $J_{0}\left(\mu_{n}\right)=0$. The moisture content $M_{t}^{s t}$ of a strut at time $t$ can be obtained by integrating $C(r, t)$ in Eq. (A-7) over the domain, see Fig. 6c, to give

$$
M_{t}^{s t}=\frac{\int_{0}^{2 \pi} \int_{0}^{R} C(r, t) \cdot r d r d \varphi}{\pi R^{2}}=M_{\infty}\left(1-4 \cdot \sum_{n=1}^{\infty} \frac{\exp \left(-D(T) \mu_{n}^{2} t / R^{2}\right)}{\mu_{n}^{2}}\right)
$$

where $R$ is the radius of the strut and $M_{\infty}$ is the saturate moisture content of the specimen at a certain temperature $T$.

\section{Appendix B. Langmuir-type diffusion model} Diffusion across face sheets

For a 1D isotropic diffusion in principle coordinate system, the governing 
equation of a Langmuir-type diffusion process is [33]

$$
\left\{\begin{array}{l}
D_{\gamma}(T) \frac{\partial^{2} n}{\partial z^{2}}=\frac{\partial n}{\partial t}+\frac{\partial N}{\partial t} \\
\frac{\partial N}{\partial t}=\gamma n-\beta N
\end{array}\right.
$$

The boundary and initial conditions for the face sheet are

$$
\left\{\begin{array}{l}
n(0, t)=n(\delta, t)=n_{\infty} \quad t \geq 0 \\
n(z, 0)=0 \quad 0<z<\delta \\
N(z, 0)=0 \quad 0 \leq z \leq \delta
\end{array}\right.
$$

Analytical solutions for $n(z, t)$ and $N(z, t)$ in Eq. (A-9) were previously provided by Carter and Kibler [33] and the total moisture concentration $C(z, t)$ in the face sheet is given by Eq. (12). Hence, the total moisture content of a face sheet $M_{t}^{f_{s}}$ is obtained by integrating $C(z, t)$ over the domain to give

$$
M_{t}^{f_{s}}=\frac{\int_{0}^{\delta} C(z, t) d z}{\delta}=M_{\infty}\left(1-\frac{8}{\pi^{2}} \sum_{s=1}^{\infty(\text { odd })} \frac{\omega_{s}^{+} e^{-\omega_{s}^{-} t}-\omega_{s}^{-} e^{-\omega_{s}^{+} t}}{s^{2}\left(\omega_{s}^{+}-\omega_{s}^{-}\right)}+\frac{8}{\pi^{2}}\left(\frac{k_{p} \beta}{\gamma+\beta}\right) \sum_{s=1}^{\infty \text { (odd) }} \frac{e^{-\omega_{s}^{-} t}-e^{-\omega_{s}^{+} t}}{\left(\omega_{s}^{+}-\omega_{s}^{-}\right)}\right)
$$

where $k_{p}$ and $\omega_{s}^{ \pm}$are

$$
\left\{\begin{array}{l}
k_{p}=\frac{\pi^{2} D_{\gamma}}{\delta^{2}} \\
\omega_{s}^{ \pm}=\frac{1}{2}\left[\left(k s^{2}+\gamma+\beta\right) \pm \sqrt{\left(k s^{2}+\gamma+\beta\right)^{2}-4 k \beta s^{2}}\right]
\end{array} .\right.
$$

\section{Diffusion across struts}

The Langmuir-type diffusion model in 1D cylinder coordinate system is given by

$$
\left\{\begin{array}{l}
D_{\gamma}(T)\left(\frac{\partial^{2} n}{\partial r^{2}}+\frac{1}{r} \frac{\partial n}{\partial r}\right)=\frac{\partial n}{\partial t}+\frac{\partial N}{\partial t} \\
\frac{\partial N}{\partial t}=\gamma n-\beta N
\end{array} .\right.
$$

Mathematically, the solution to Eq. (A-13) can be obtained by replacing the trigonometrical series in Eq. (A-13) with a series of Bessel functions. To obtain an exact analytical solution, the Laplace transform is employed. The boundary and initial conditions are similar to Eq. (A-6), where only the concentration of mobile molecules on the boundary is considered, as follows:

$$
\left\{\begin{array}{l}
n(R, t)=n_{\infty} ; n(0, t)<\infty \quad t \geq 0 \\
n(r, 0)=0 \quad 0 \leq r<R \\
N(r, 0)=0 \quad 0 \leq r \leq R
\end{array} .\right.
$$

Taking the Laplace transform of $n(r, t)$ and $N(r, t)$ with respect to time $t$, Eq. (A-13) can be re-expressed in the form of

$$
\frac{d^{2} \bar{n}(r, p)}{d r^{2}}+\frac{1}{r} \frac{d \bar{n}(r, p)}{d r}=\frac{1}{D_{\gamma}}(p \bar{n}(r, p)+p \bar{N}(r, p))
$$




$$
p \bar{N}(r, p)=\gamma \bar{n}(r, p)-\beta \bar{N}(r, p)
$$

where $\bar{n}(r, p)$ and $\bar{N}(r, p)$ are the Laplace transformation of $n(r, t)$ and $N(r, t)$, respectively. From Eq. (A-14), the corresponding boundary conditions are

$$
\bar{n}(R, p)=n_{\infty} / p ; \bar{n}(0, p)<\infty \quad t \geq 0 \quad \text {. }
$$

Substituting Eq. (A-16) into (A-15), the latter can be rewritten in form of a Bessel equation

$$
\frac{d^{2} \bar{n}}{d r^{2}}+\frac{1}{r} \frac{d \bar{n}}{d r}-\lambda^{2} \bar{n}=0
$$

where

$$
\lambda=\sqrt{\frac{1}{D_{\gamma}}\left(\frac{p \gamma}{p+\beta}+p\right)} .
$$

The solution to Eq. (A-18), subject to the boundary conditions of Eq. (A-17), is

$$
\bar{n}(r, p)=\frac{n_{\infty}}{p} \frac{I_{0}(\lambda r)}{I_{0}(\lambda R)}
$$

where $I_{0}$ is the modified Bessel function of the first kind of order zero. From Eq. (A-16), one obtains

$$
\bar{N}(r, p)=\left(\frac{\gamma}{p+\beta}\right) \frac{n_{\infty}}{p} \frac{I_{0}(\lambda r)}{I_{0}(\lambda R)}
$$

The concentration of bound molecules $N(r, t)$ is the inverse Laplace transform of Eq. (A-21) given by

$$
N(r, t)=\frac{1}{2 \pi i} \int_{\eta-i \infty}^{\eta+i \infty} \bar{N}(r, p) e^{p t} d p
$$

where $\eta$ is an arbitrary positive number and $p=\eta+i q$. Evaluation of Eq. (A-22) by the residue theory gives [43]

$$
N(r, t)=\frac{1}{2 \pi i} \int_{s-i \infty}^{s+i \infty} \bar{N}(r, p) e^{p t} d p=\sum_{n=1}^{\infty} \operatorname{RES}\left[\bar{N}(r, p) e^{p t}, s_{n}\right]
$$

where $s_{n}$ are the poles of $\bar{N}(r, p)$. The points $(\eta=-(\gamma+\beta), q=0)$, $(\eta=-\beta, q=0),(\eta=0, q=0)$ and two infinite sets of points $\left(\eta=\psi_{n}^{ \pm}, q=0\right)$ are the poles of $\bar{N}(r, p)$ where

$$
\psi_{n}^{ \pm}=\frac{1}{2}\left[-\left(\mu_{n}^{2} k_{c}+\gamma+\beta\right) \pm \sqrt{\left(\mu_{n}^{2} k_{c}+\gamma+\beta\right)^{2}-4 \mu_{n}^{2} k_{c} \beta}\right]
$$

and $\mu_{n}$ are roots of the equation $J_{0}\left(\mu_{n}\right)=0 . k_{c}$ is defined as

$$
k_{c}=D_{\gamma} / R^{2}
$$

From Eq. (A-23), one obtains the concentration of bound moisture molecules

$$
N(r, t)=\frac{n_{\infty} \gamma}{\beta}\left(1+2 \sum_{n=1}^{\infty} \frac{J_{0}\left(\mu_{n} r / R\right)}{J_{1}\left(\mu_{n}\right) \cdot \mu_{n}} \frac{\left(\psi_{n}^{-} e^{\psi_{n}^{+} t}-\psi_{n}^{+} e^{\psi_{n}^{-} t}\right)}{\left(\psi_{n}^{+}-\psi_{n}^{-}\right)}\right)
$$

From Eqs. (A-13b) and (A-26), the concentration of mobile moisture molecules is 


$$
\begin{aligned}
n(r, t) & =\frac{n_{\infty}}{\beta} \sum_{n=1}^{\infty} \frac{2 J_{0}\left(\mu_{n} r / R\right)}{J_{1}\left(\mu_{n}\right) \cdot \mu_{n}} \frac{\psi_{n}^{+} \psi_{n}^{-}\left(e^{\psi_{n}^{+} t}-e^{\psi_{n}^{-} t}\right)}{\left(\psi_{n}^{+}-\psi_{n}^{-}\right)} \\
& +n_{\infty}\left(1+\sum_{n=1}^{\infty} \frac{2 J_{0}\left(\mu_{n} r / R\right)}{J_{1}\left(\mu_{n}\right) \cdot \mu_{n}} \frac{\left(\psi_{n}^{-} e^{\psi_{n}^{+} t}-\psi_{n}^{+} e^{\psi_{n}^{-} t}\right)}{\left(\psi_{n}^{+}-\psi_{n}^{-}\right)}\right)
\end{aligned}
$$

The moisture content of a strut $M_{t}^{s t}$ is obtained by integrating $C(r, t)$ over the domain to give

$$
\begin{aligned}
M_{t}^{s t} & =\int_{0}^{2 \pi} \int_{0}^{R} C(r, t) \cdot r d r d \varphi / \pi R^{2} \\
& =\left(\int_{0}^{2 \pi} \int_{0}^{R} n(r, t) \cdot r d r d \varphi+\int_{0}^{2 \pi} \int_{0}^{R} N(r, t) \cdot r d r d \varphi\right) / \pi R^{2} .
\end{aligned}
$$

Substituting Eqs. (A-26) and (A-27) into (A-28), one obtains the moisture content of a single strut as follows:

$$
M_{t}^{s t}=M_{\infty}\left(1-4 \sum_{n=1}^{\infty} \frac{\left(\zeta_{n}^{+} e^{-\zeta_{n}^{-} t}-\zeta_{n}^{-} e^{-\zeta_{n}^{+} t}\right)}{\mu_{n}^{2}\left(\zeta_{n}^{+}-\zeta_{n}^{-}\right)}+4\left(\frac{k_{c} \beta}{\gamma+\beta}\right) \sum_{n=1}^{\infty} \frac{\left(e^{-\zeta_{n}^{-} t}-e^{-\zeta_{n}^{+} t}\right)}{\left(\zeta_{n}^{+}-\zeta_{n}^{-}\right)}\right)
$$

where

$$
\zeta_{n}^{ \pm}=\frac{1}{2}\left(\mu_{n}^{2} k_{c}+\gamma+\beta \pm \sqrt{\left(\mu_{n}^{2} k_{c}+\gamma+\beta\right)^{2}-4 \mu_{n}^{2} k_{c} \beta}\right) .
$$

\section{Appendix C. Finite element modelling of Langmuir-type diffusion}

The subroutine HETVAL can only be used in ABAQUS heat transfer analysis. Hence, by making the analogy of diffusion with heat transfer, one can convert the diffusion parameters to heat transfer parameters. The govern equation of classical Fick diffusion is given in Eq. (36). The govern equation of $1 \mathrm{D}$ heat transfer is given as

$$
\int_{V}\left(\frac{d T}{d t}+\frac{\partial}{\partial x} \cdot \mathbf{J}_{T}\right) d V=0
$$

where $\mathbf{J}_{T}$ is the heat flux and defined as

$$
\mathbf{J}_{T}=-\left(\frac{k_{T}}{c \rho_{m}}\right) \cdot\left(\frac{\partial T}{\partial x}\right)
$$

where $k_{T}$ is the thermal conductivity, $c$ is the specific heat and $\rho_{m}$ is the material density. By comparing Eq. (36) and Eq. (A-31), diffusion can be modeled by equating $D_{\gamma}$ with $k_{T}$ and $C$ with $T$ when the values of $c$ and $\rho_{m}$ are unit.

In the first of Eq. (A-9), the bound water molecules of concentration $N$ can be seen as the 'sinks' of mobile water molecules and $\partial N / \partial t$ is the flux of the transform process. The user subroutine HETVAL is available for introducing a heat flux due to internal heat generation in a material. A negative flux such as $-\partial N / \partial t$ can be introduced by HETVAL to model the 'sinks' of mobile water molecules. The concentration of bound water molecules $N$ at every material calculation point is needed to provide the flux $-\partial N / \partial t$ from the second of Eq. (A-9) and is given as

$$
\mathbf{J}_{N(i)}=\gamma n_{i}-\beta N_{i}
$$

where $n_{i}$ and $N_{i}$ are the concentration of mobile and bound water molecules at the 
beginning of $i$-th increment, respectively. $\mathbf{J}_{N(i)}$ is the flux of $N$ at the beginning of $i$-th increment. The values of $N_{i}$ are maintained during the analysis by passing into the user-defined solution-dependent state variable (SDV). $N_{i}$ are calculated by implicit Euler method from the second of Eq. (22) given by

$$
N_{i}=N_{i-1}+\frac{1}{2} \Delta t_{i-1}\left(\overline{\mathbf{J}}_{N(i)}+\mathbf{J}_{N(i-1)}\right)
$$

where $\Delta t_{i-1}$ is the time increment of $(i-1)$-th increment. $\overline{\mathbf{J}}_{N(i)}$ is given as

$$
\mathbf{J}_{N(i-1)}=\gamma n_{i-1}-\beta N_{i-1}
$$

and

$$
\overline{\mathbf{J}}_{N(i)}=\gamma n_{i}-\beta\left(N_{i-1}+\Delta t_{i-1} \cdot \mathbf{J}_{N(i-1)}\right)
$$

The output of concentration of mobile water molecules $n$ and the concentration of bound water molecules $N$ are respectively obtained by FEM. To gain the total moisture concentration $C$ at the finite element nodes and total moisture content $M_{t}$ of the model, a Python code is adopted to calculate the sum of moisture concentration $n$ and $N . M_{t}$ can be obtained by integrating concentration $C$ over the whole model.

\section{Acknowledgement}

The present work is supported by National Science Foundation of China under Grant Nos. 11402094 and 11802100, the Fundamental Research Funds for the Central Universities under Grant No. HUST: 2018KFYYXJJ010.

\section{References}

[1] V.V. Vasiliev, V.A. Barynin, A.F. Razin, Anisogrid composite lattice structures -Development and aerospace applications, Compos Struct. 94 (2012) 1117-1127.

[2] G.D. Bella, L. Calabrese, C. Borsellino, Mechanical characterisation of a glass/polyester sandwich structure for marine applications, Mater Des. 42 (2012) 486-494.

[3] A.G. Evans, J.W. Hutchinson, N.A. Fleck, M.F. Ashby, H.N.G. Wadley, The topological design of multifunctional cellular metals, Pro Mater Sci. 46 (2001) 309-327.

[4] H.L. Fan, D.N. Fang, L.M. Chen, Z. Dai, W. Yang, Manufacturing and testing of a CFRC sandwich cylinder with Kagome cores, Compos Sci Technol. 69 (15-16) (2009) 2695-2700.

[5] H.L. Fan, L. Zhao, H.L. Chen, N. Kuang, C.K. Yang, S.Q. Huang, Y.F. Jiang, Ductile deformation mechanisms and designing instructions for integrated woven textile sandwich composites, Compos Sci Technol. 72 (2012) 1338-1343.

[6] Y. Hu, W.X. Li, X.Y. An, H.L. Fan, Fabrication and mechanical behaviors of corrugated lattice truss composite sandwich panels, Compos Sci Technol. 125 (2016) 114-122.

[7] J. Xiong, L. Ma, L.Z. Wu, A. Vaziri, J. Yang, L. Wu, Mechanical behavior of carbon fiber composite lattice core sandwich panels fabricated by laser cutting, Acta Mater. 60 (13-14) (2012) 5322-5334.

[8] J. Xiong, R. Ghosh, L. Ma, A. Vaziri, Y.L. Wang, L.Z. Wu, Sandwich-walled cylindrical shells with lightweight metallic lattice truss cores and carbon fiber-reinforced composite face sheets, Compos Part A Appl S. 56 (1) (2014) 
226-238.

[9] J. Xiong, L. Ma, S.D. Pan, L.Z. Wu, J. Papadopoulos, A. Vaziri, Shear and bending performance of carbon fiber composite sandwich panels with pyramidal truss cores, Acta Mater. 60 (4) (2012) 1455-1466.

[10] J.S. Yang, L. Ma, K.U. Schröderc, Y.L. Chen, S. Lia, L.Z. Wu, R. Schmid, Experimental and numerical study on the modal characteristics of hybrid carbon fiber composite foam filled corrugated sandwich cylindrical panels, Polym Test. 68 (2018) 8-18.

[11] K. Finnegan, G. Kooistra, H.N.G. Wadley, V.S. Deshpande, The compressive response of carbon fiber composite pyramidal truss sandwich cores, Int J Mater Res. 98 (12) (2007) 1264-1272.

[12] G. Qi, L. Ma, Experimental investigation of composite pyramidal truss core sandwich panels with lightweight inserts, Compos Struct. 187 (2018) 336-343.

[13] M. Subhani, A. Al-Ameri, Strength reduction in square columns confined with CFRP under marine environment, Compos B Eng. 97 (2016) 183-92.

[14] L. Mansouri, A. Djebbar, S. Khatir, M.A. Wahab, Effect of hygrothermal aging in distilled and saline water on the mechanical behaviour of mixed short fibre/woven composites, Compos Struct. 207 (2019) 816-825.

[15] F. Tian, Z. Zhong, Y. Pan, Modeling of natural fiber reinforced composites under hygrothermal ageing, Compos Struct. 200 (2018) 144-152.

[16] N. Tual, N. Carrere, P. Davies, T. Bonnemains, E. Lolive, Characterization of sea water ageing effects on mechanical properties of carbon/epoxy composites for tidal turbine blades, Compos Part A Appl S. 78 (2015) 380-389.

[17] M. Arhant, P.Y.L. Gac, M.L. Gall, C. Burtin, C. Criancon, P. Davies, Effects of sea water and humidity on the tensile and compresisve properties of carbon-polyamide 6 laminates, Compos Part A Appl S. 91 (2016) 250-261.

[18] L.S. Sutherland, A review of impact testing on marine composite materials: Part III - Damage tolerance and durability, Compos Struct. 188 (2018) 512-518.

[19] Y.I. Tsai, E.J. Bosze, E. Barjasteh, S.R. Nutt, Influence of hygrothermal environment on thermal and mechanical properties of carbon fiber/fiberglass hybrid composites, Compos Sci Technol. 69 (2009) 432-437.

[20] D. D. Macdonald. The Mathematics of Diffusion. 1956.

[21] C.H. Shen, G.S. Springer, Moisture absorption and desorption of composite materials, Compos Mater. 10 (1) (1976) 2-20.

[22] D.A. Bond. Moisture diffusion in a fiber-reinforced composite: Part I non-Fickian transport and the effect of fiber spatial distribution, J Compos Mater. 39 (2005) 2113-2141.

[23] L.R. Grace, M.C. Altan, Characterization of anisotropic moisture absorption in polymeric composites using hindered diffusion model, Compos Part A Appl S. 43 (2012) 1187-1196.

[24] J.Z. Wang, F.H. Dai, L. Ma, A multi-scale moisture diffusion coupled with stress model for composite materials, Compos Struct. 171 (2017) 345-359.

[25] S. Roy, W.X. Xu, S.J. Park, K.M. Liechti, Anomalous Moisture Diffusion in Viscoelastic Polymers: Modeling and Testing, J. Appl. Mech. 67(2) (1999) 391-396.

[26] ASTM: D5229 Standard test method for moisture absorption properties and equilibrium conditioning of polymer matrix composite materials.

[27] N. Guermazi, A.B. Tarjem, I. Ksouri, H.F. Ayedi, On the durability of FRP composites for aircraft structures in hygrothermal conditioning, Compos Part B Eng. 85 (2016) 294-304. 
[28] ASTM: E2954-15 axial compression test of reinforced plastic and polymer matrix composite vertical members.

[29] ASTM: C365-16 standard test method for flatwise compressive properties of sandwich cores.

[30] Y.I. Tsai, E.J. Bosze, E. Barjasteh, S.R. Nutt, Influence of hygrothermal environment on thermal and mechanical properties of carbon fiber/fiberglass hybrid composites, Compos Sci Technol. 69 (3-4) (2009) 432-437.

[31] P. Vaddadi, T. Nakamura, R.P. Singh, Transient hygrothermal stresses in fiber reinforced composites: a heterogeneous characterization approach, Compos Part A Appl S. 34 (2003) 719-730.

[32] V.M. Karbhari, G. Xian, Hygrothermal effects on high VF pultruded unidirectional carbon/epoxy composites: Moisture uptake, Compos Part B Eng. 40 (1) (2009) 41-49.

[33] H.G. Carter, K.G. Kibler, Langmuir-type model for anomalous moisture diffusion in composite resins, J Compos Mater. 12 (2) (1978) 118-131.

[34] W.K. Loh, A.D. Crocombe, M.M.A. Wahab, I.A. Ashcroft, Modelling anomalous moisture uptake, swelling and thermal characteristics of a rubber toughened epoxy adhesive, Int J Adhes Adhes. 25 (1) (2005) 1-12.

[35] A. Mubashar, I.A. Ashcroft, G.W. Critchlow, A.D. Crocombe, Modelling cyclic moisture uptake in an epoxy adhesive, J Adhesion. 85 (10) (2009) 711-735.

[36] M. Barink, A. Mavinkurve, J. Janssen, Predicting non-fickian moisture diffusion in EMCs for application in micro-electronic devices, Microelectron Reliab. 62 (2016) 45-49.

[37] B. Dao, J.H. Hodgkin, J. Krstina, J. Mardel, W. Tian, Accelerated ageing versus realistic ageing in aerospace composite materials. IV. Hot/wet ageing effects in a low temperature cure epoxy composite, J Appl Polym Sci. 106 (6) (2007) 4264-4276.

[38] E. Guzmán, J. Cugnoni, T. Gmür, Multi-factorial models of a carbon fibre/epoxy composite subjected to accelerated environmental ageing, Compos Struct. 111 (2014) 179-192.

[39] Z. Ullah, L. Kaczmarczyk, S.A. Grammatikos, M.C. Evernden, Multi-scale computational homogenisation to predict the long-term durability of composite structures, Comput Struct. 181 (2017) 21-31.

[40] ABAQUS 6.14 Help Document, User subroutine to define a material's mechanical behavior. ABAQUS User Subroutine Reference Guide.

[41] A. Zafar, F. Bertocco, J. Schjødt-Thomsen, J.C. Rauhe, Investigation of the long term effects of moisture on carbon fibre and epoxy matrix composites, Compos Sci Technol. 72 (2012) 656-666.

[42] S. Selzer, K. Friedrich, Mechanical properties and failure behaviour of carbon fibre-reinforced polymer composites under the influence of moisture, Compos Part A Appl S. 28 (6) (1997) 595-604.

[43] R.V. Churchill, J.W. Brown, Complex variables and applications. 


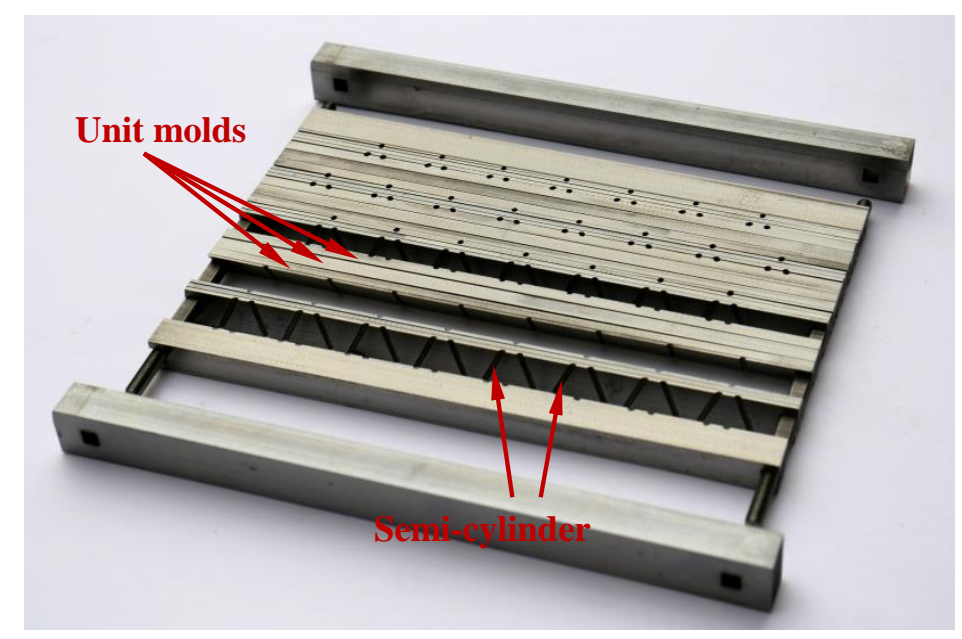

Fig. 1. The mold used to fabricate the tetrahedral truss cores sandwich panel.

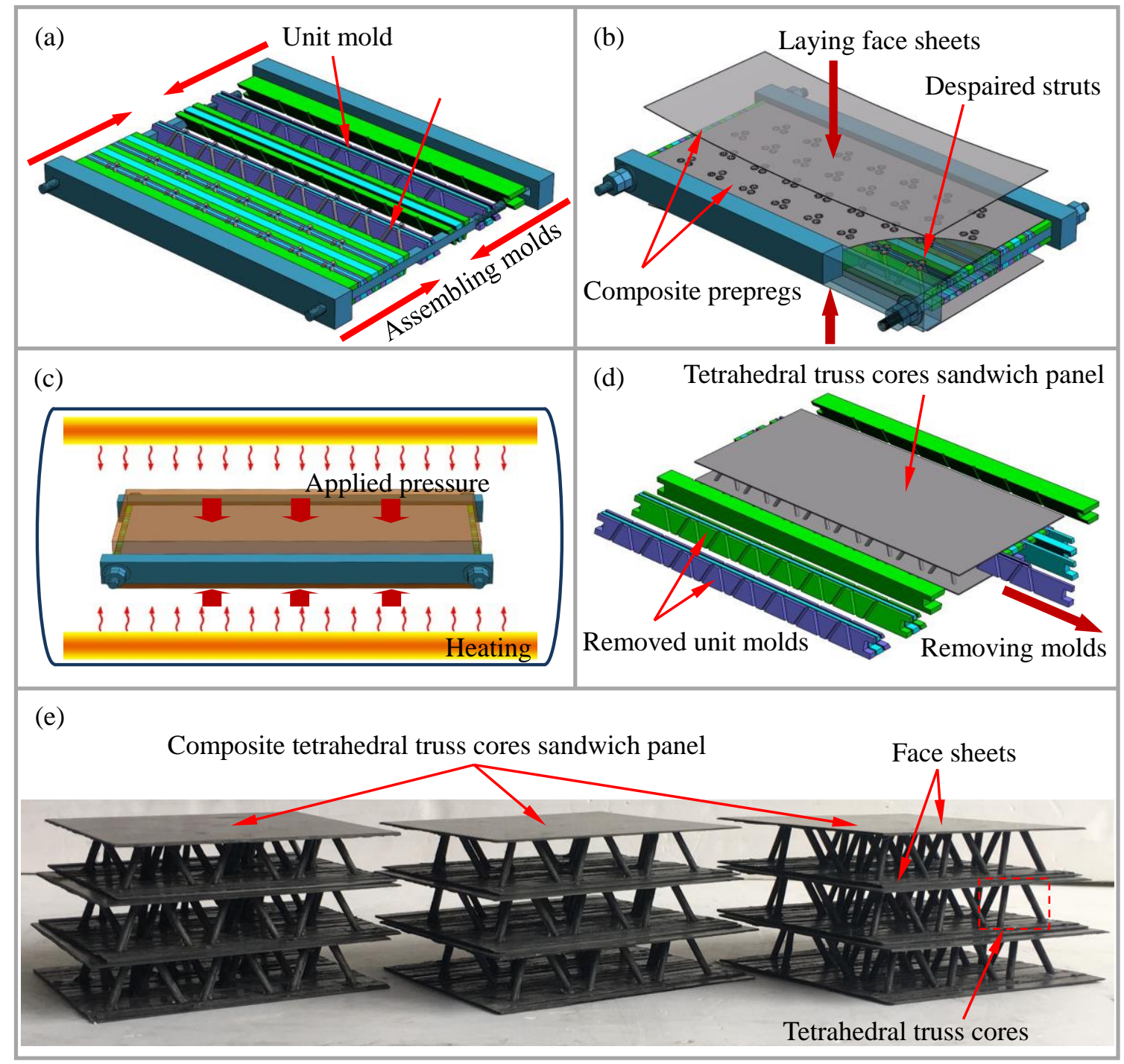

Fig. 2. Schematic for the fabrication of the CFRP tetrahedral truss cores sandwich panel: (a) assembling the composite struts; (b) laying face sheets; (c) curing process; (d) removing molds; (e) the fabricated tetrahedral truss cores sandwich panels. 

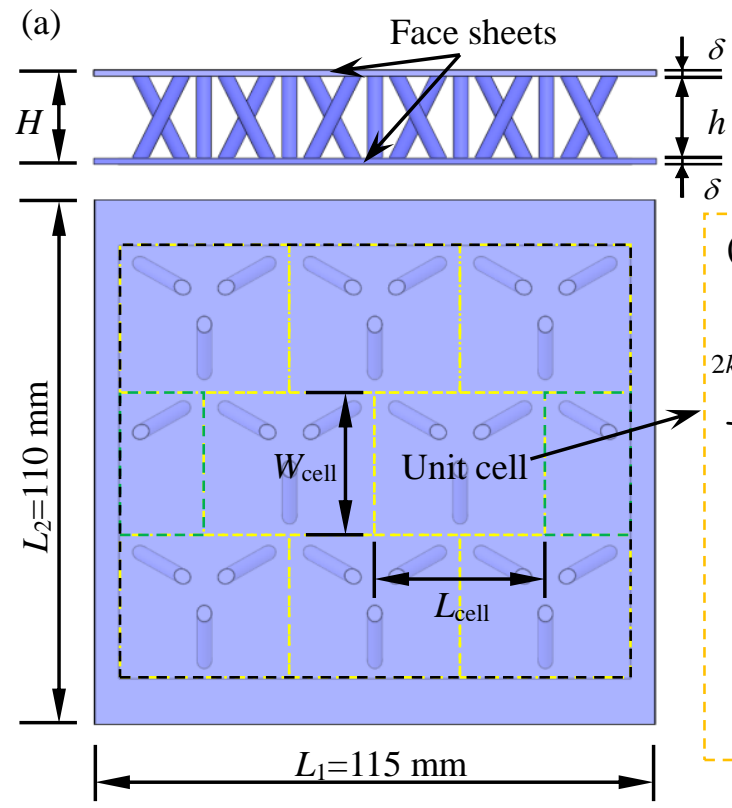

(b)

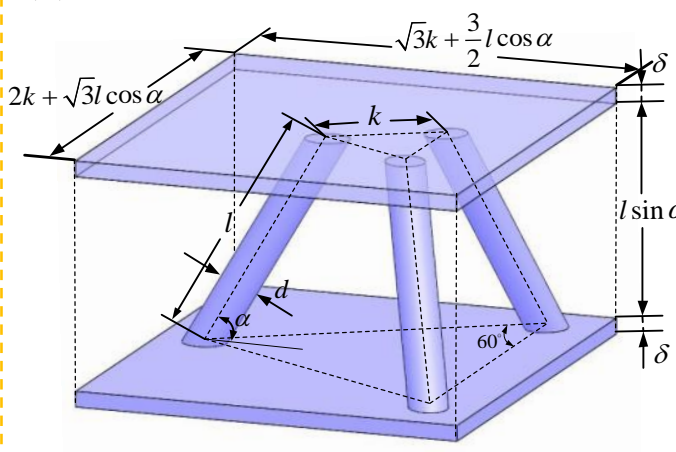

Fig. 3. (a) Dimesions of specimens used in hygrothermal experiments; (b) unit cell for a tetrahedral truss cores sandwich panel.
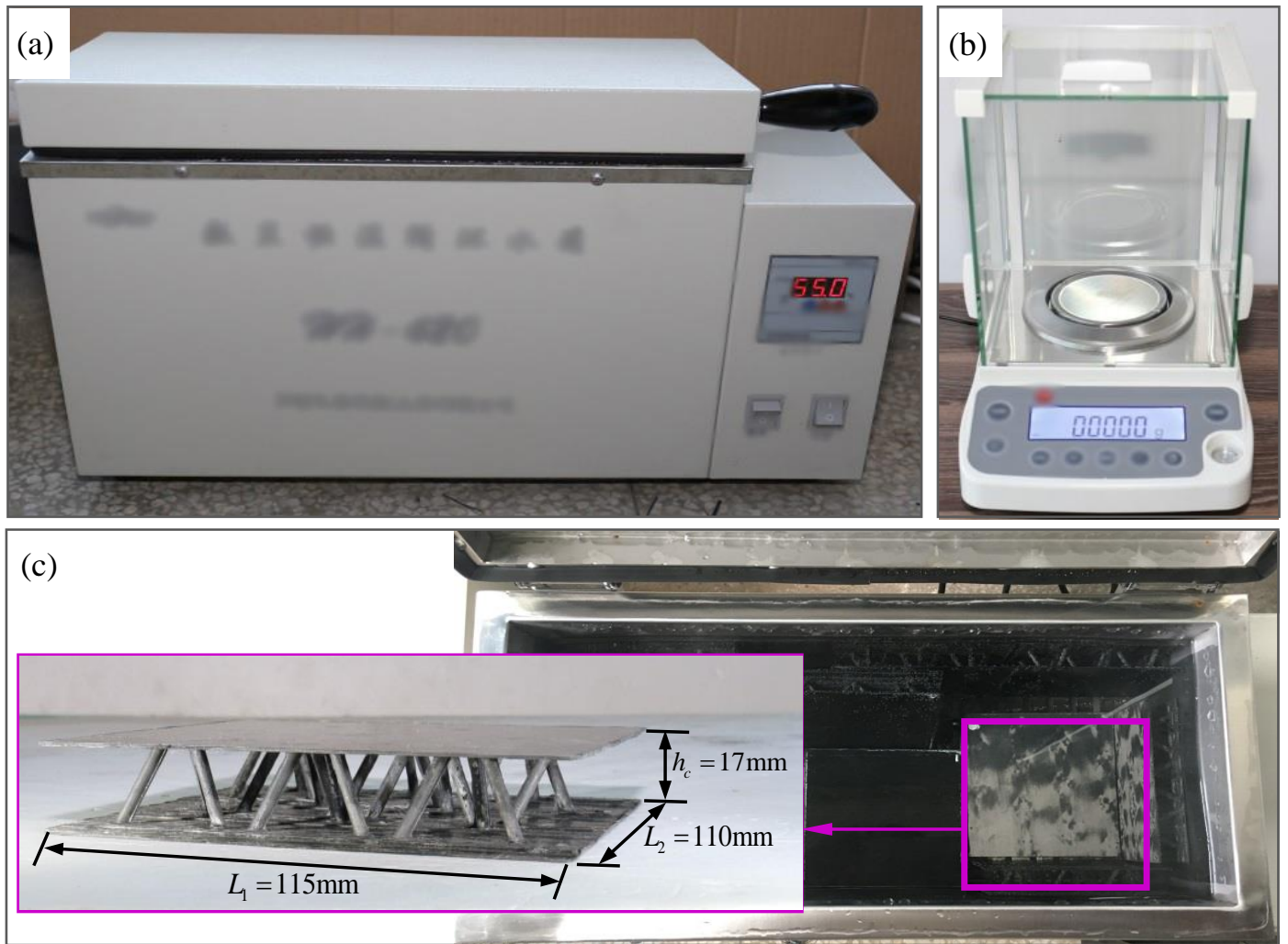

Fig. 4. (a) Water bath ; (b) electronic balance; (c) typical tetrahedral truss core sandwich panel used in a moisture absorption test. 

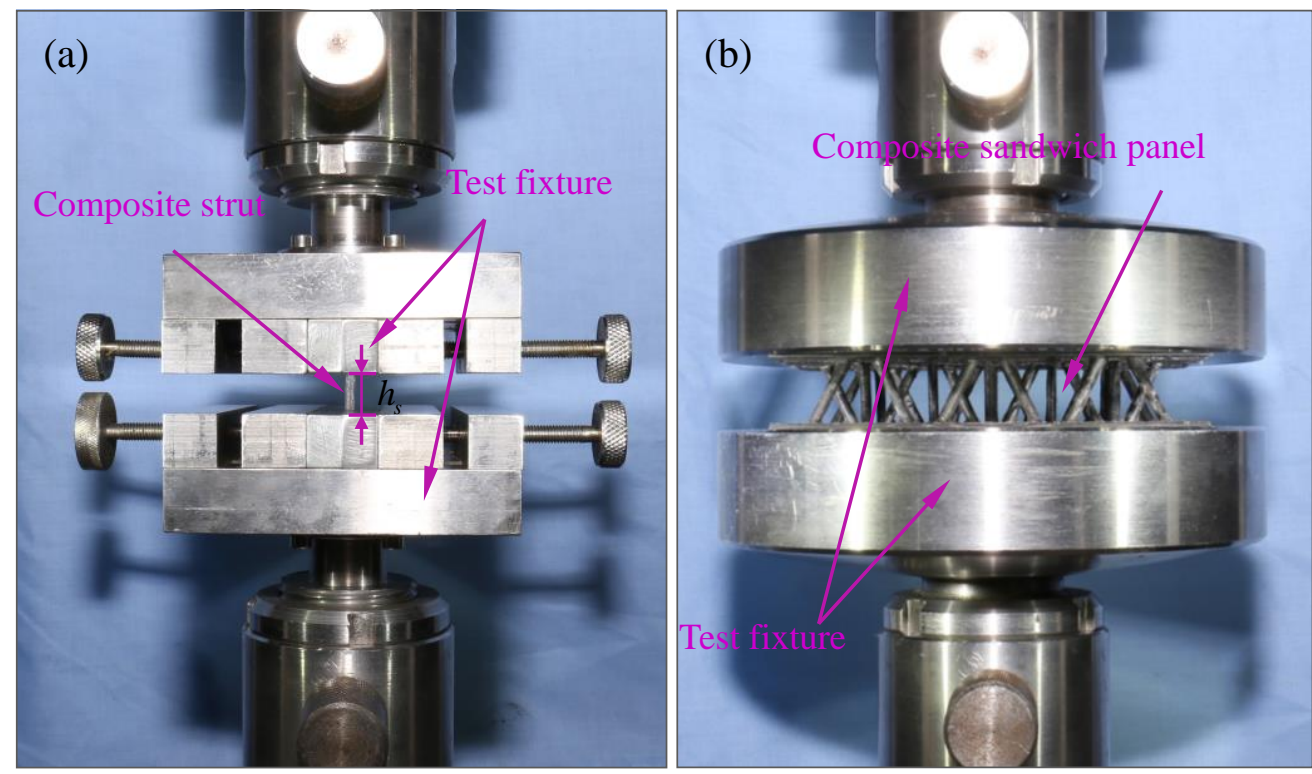

Fig. 5. Compression testing of (a) composite strut and (b) tetrahedral truss cores sandwich panel.

(a)

Surfaces exposed to

hygrothermal environment

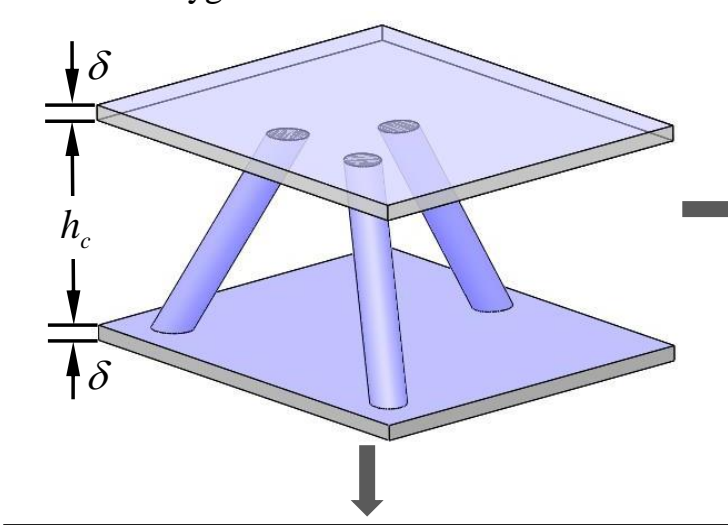

(c)

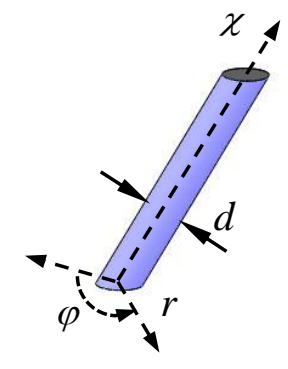

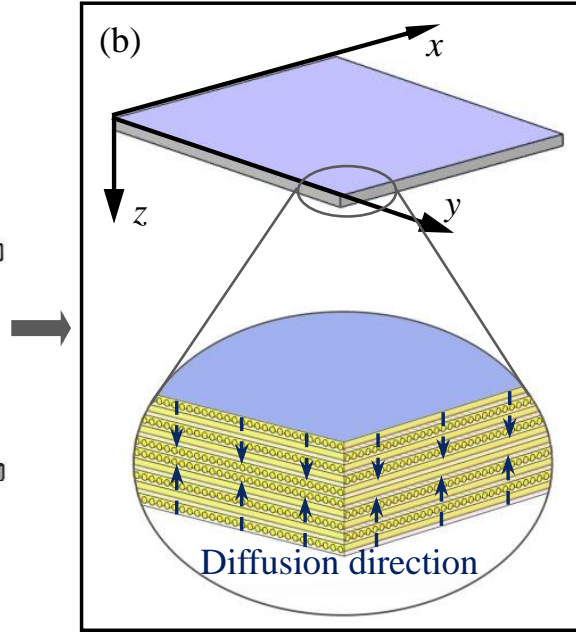

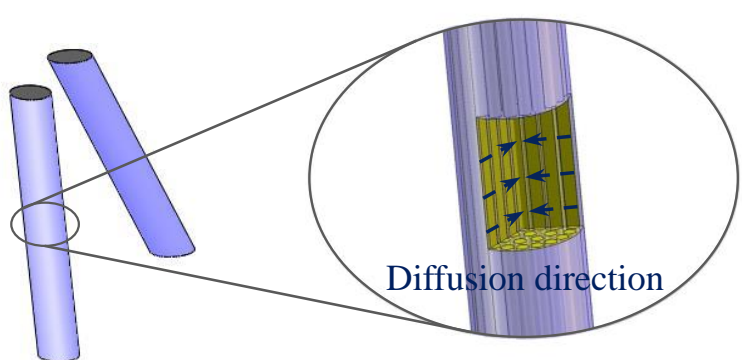

Fig. 6. (a) Surfaces in the unit cell that are exposed to moisture absorption; and direction of moisture diffusion in (b) face sheet, (c) composite strut considered by the analytical model. 


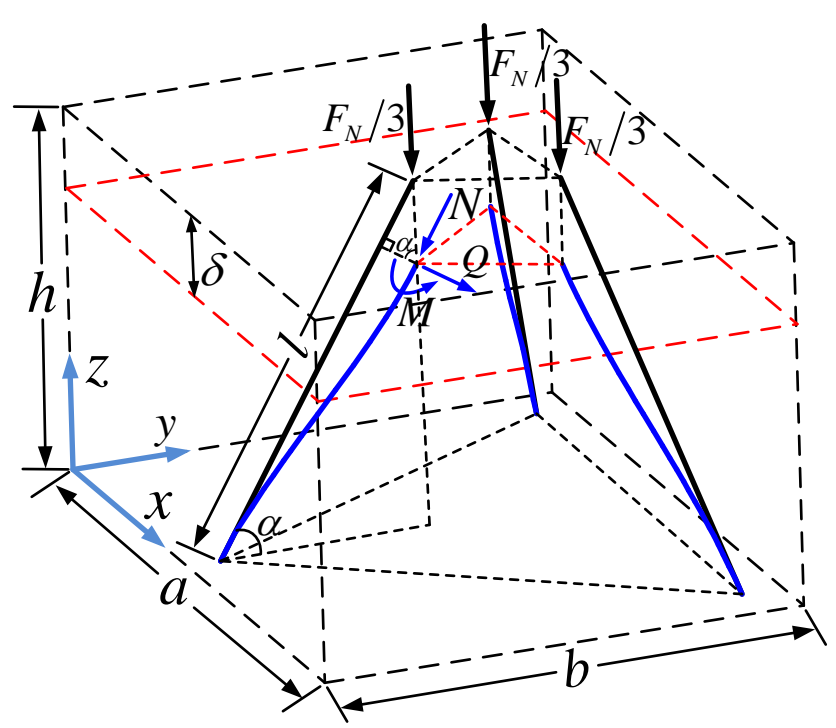

Fig. 7. Compressive deformation of the tetrahedral truss core (shown here for a unit cell).

(a)

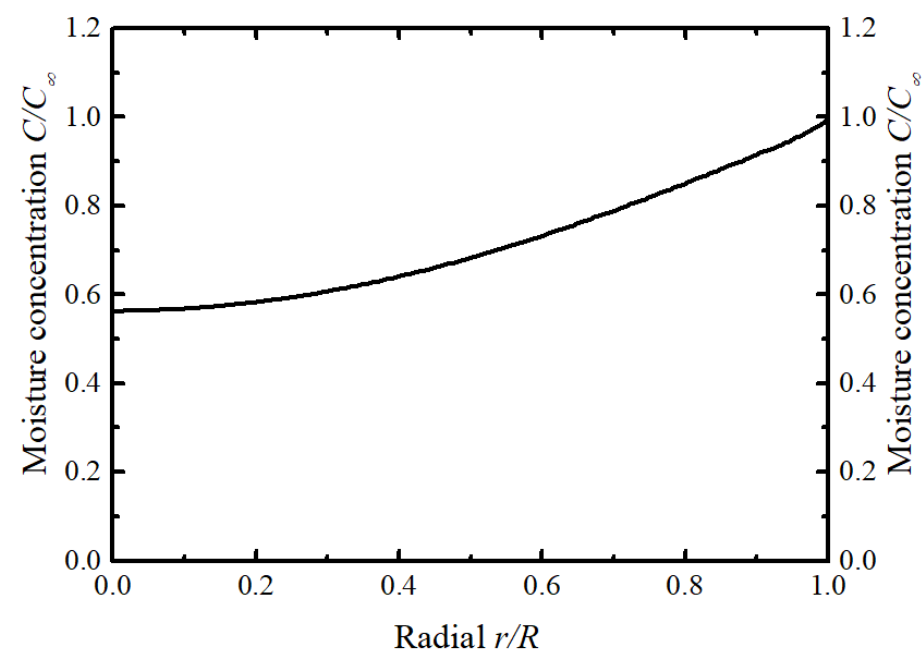

(b)

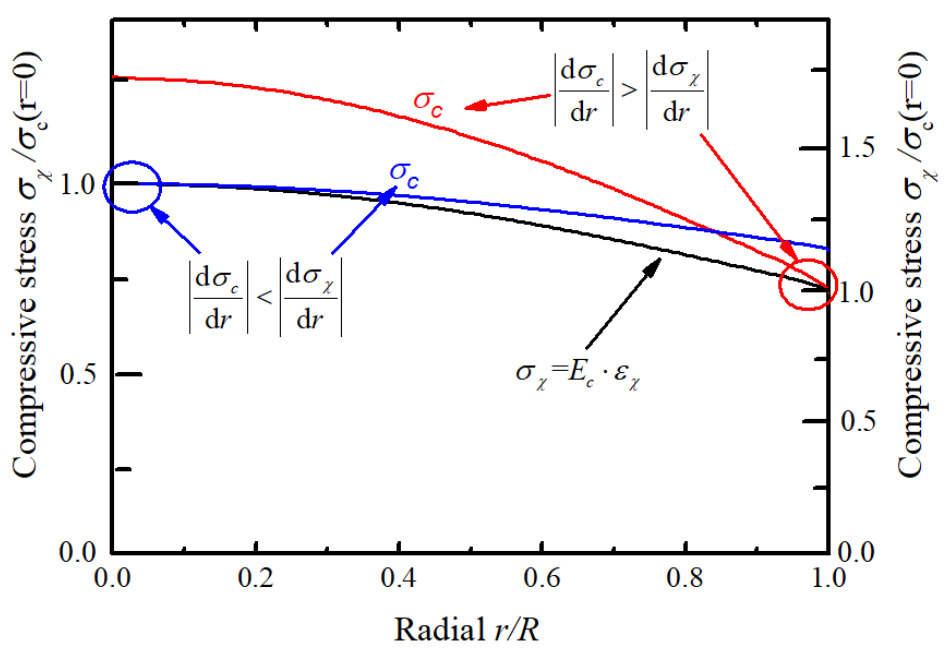

Fig. 8. (a) Typical moisture concentration distribution in a strut; (b) two cases of the site where initial failure occur: the center of the strut and the surface of the strut. 


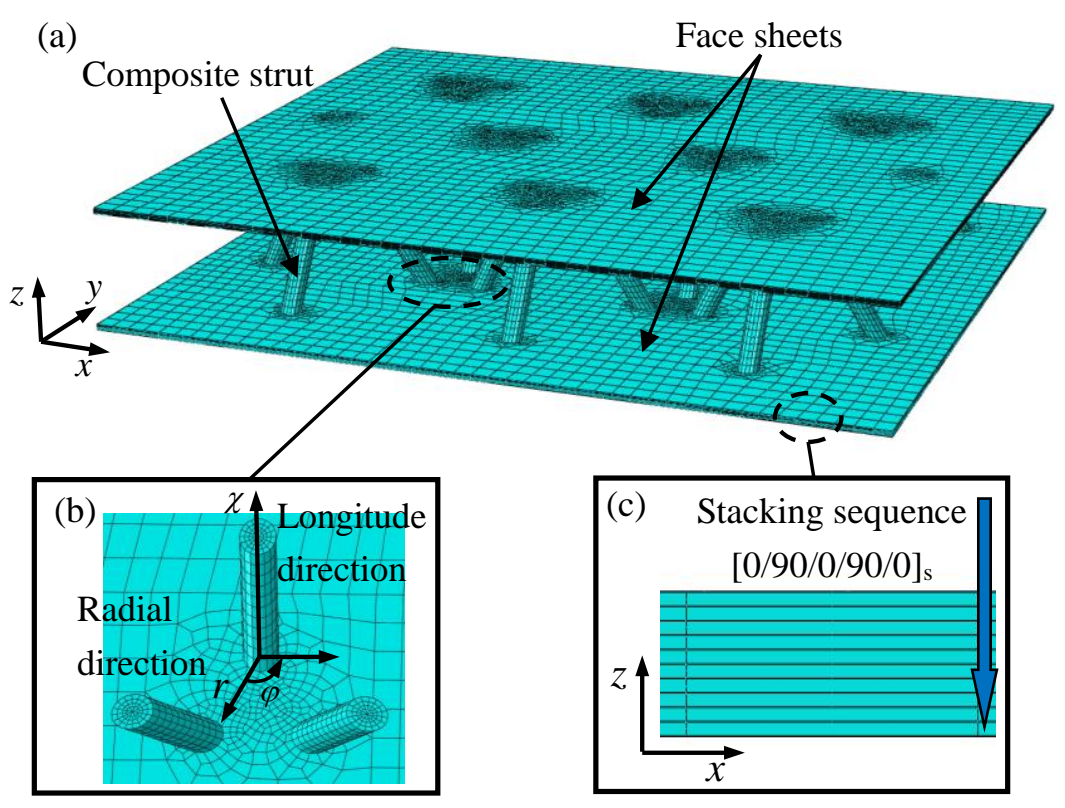

Fig. 9. (a) FEM model of tetrahedral truss cores sandwich panel; (b) the local cylinder coordinate system of the tetrahedral truss cores; (c) the local rectangular coordinate system and stacking sequence of the face sheets.

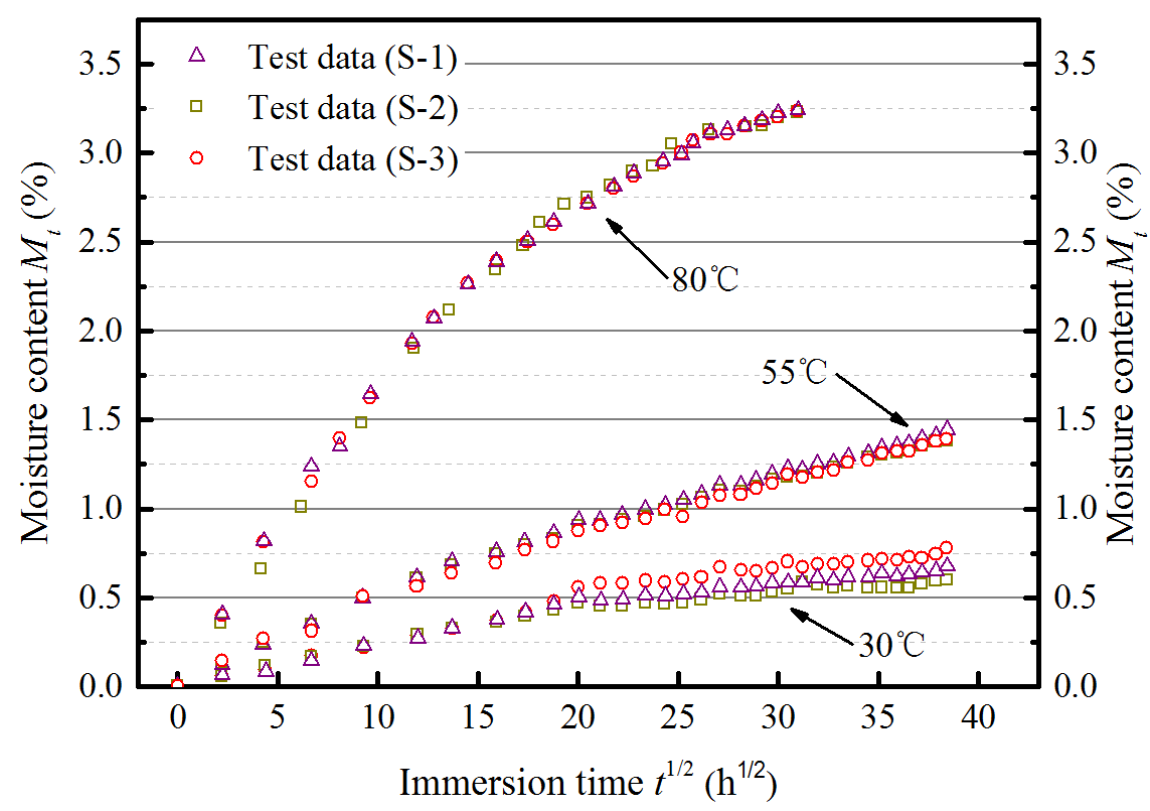

Fig. 10. Typical moisture diffusion curves measured at $30^{\circ} \mathrm{C}, 55^{\circ} \mathrm{C}$ and $80^{\circ} \mathrm{C}$. 


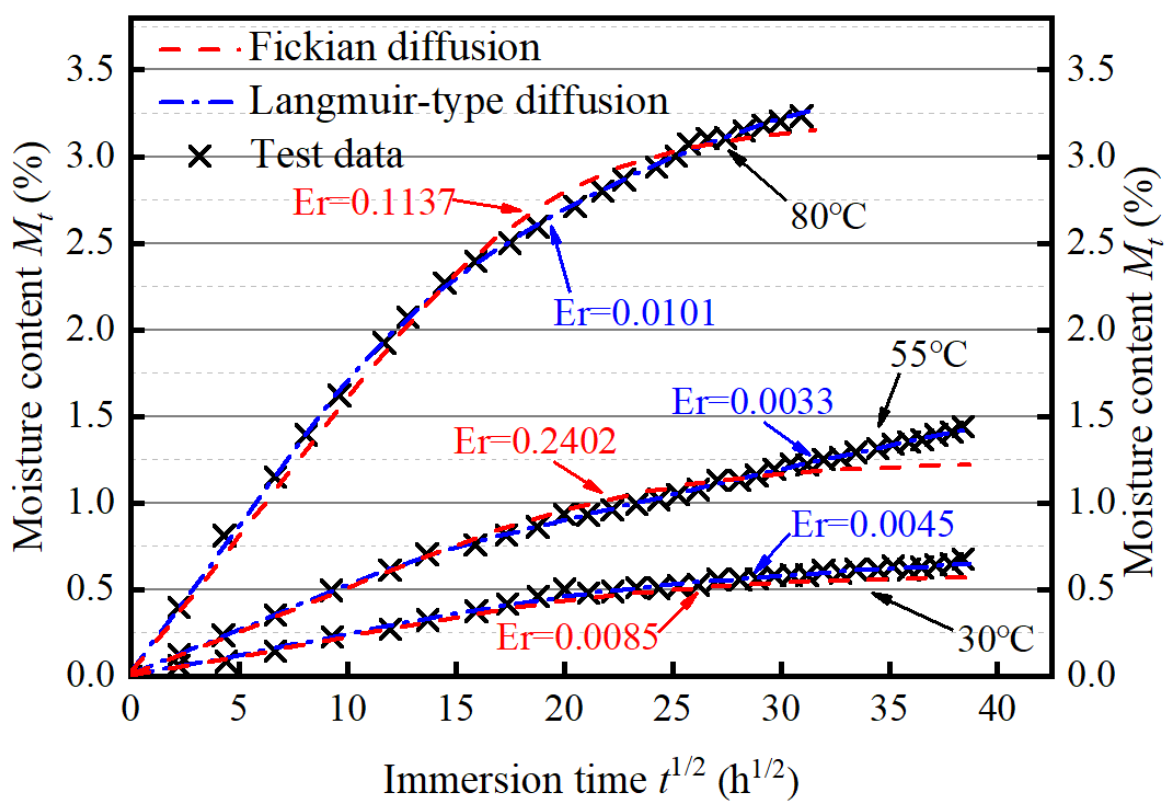

Fig. 11. Experimental data and fitted moisture diffusion curves at $30^{\circ} \mathrm{C}, 55^{\circ} \mathrm{C}$ and $80^{\circ} \mathrm{C}$ (Er is the regression residual).
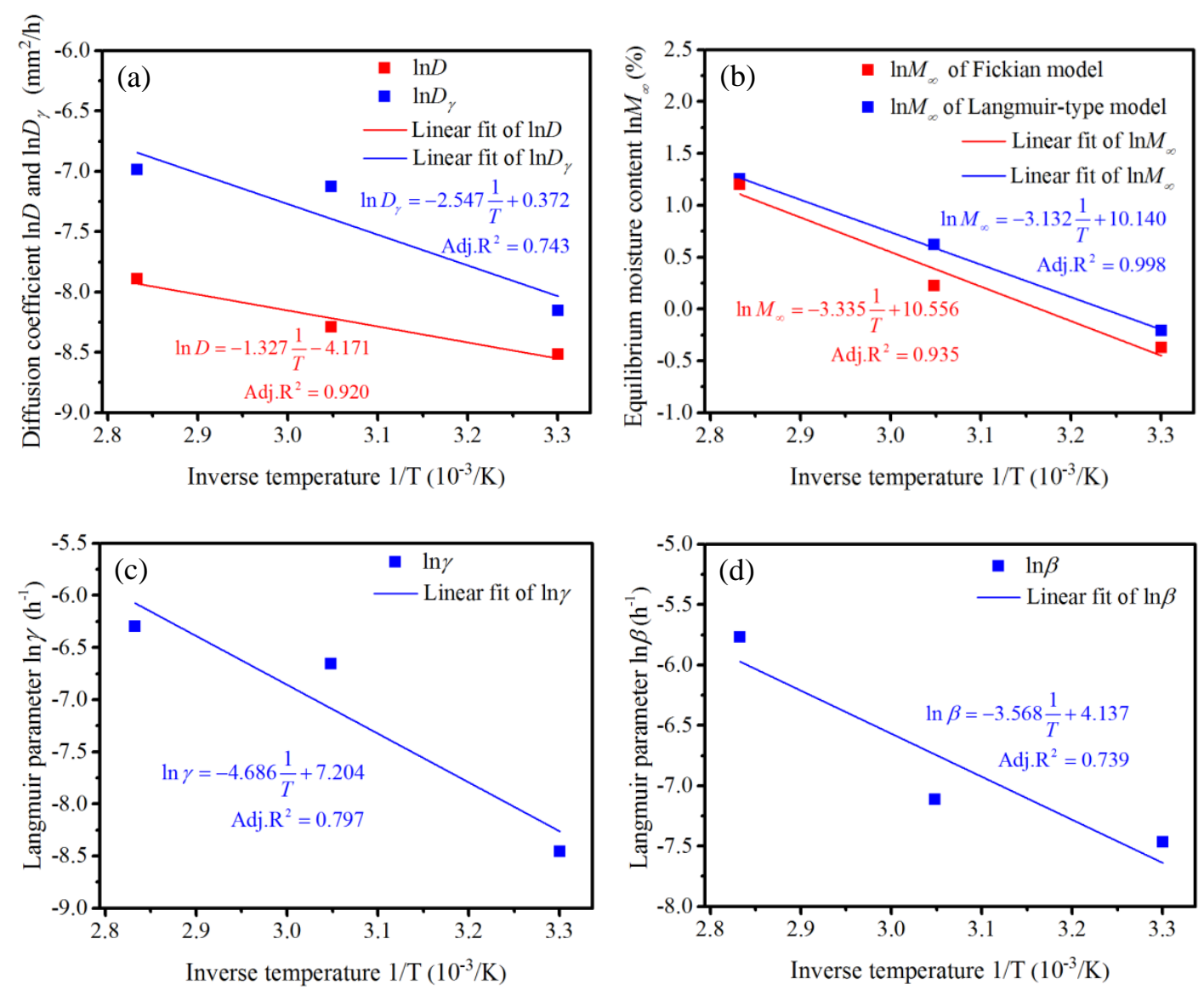

Fig. 12. Graph of Arrhenius equation: (a) $\ln D$ and $\ln D_{\gamma}$ against inverse temperature 1/T; (b) $\ln M_{\infty}$ against inverse temperature $1 / \mathrm{T}$. (c) $\ln \gamma$ against inverse temperature $1 / \mathrm{T}$; (d) $\ln \beta$ against inverse temperature $1 / \mathrm{T}$. 

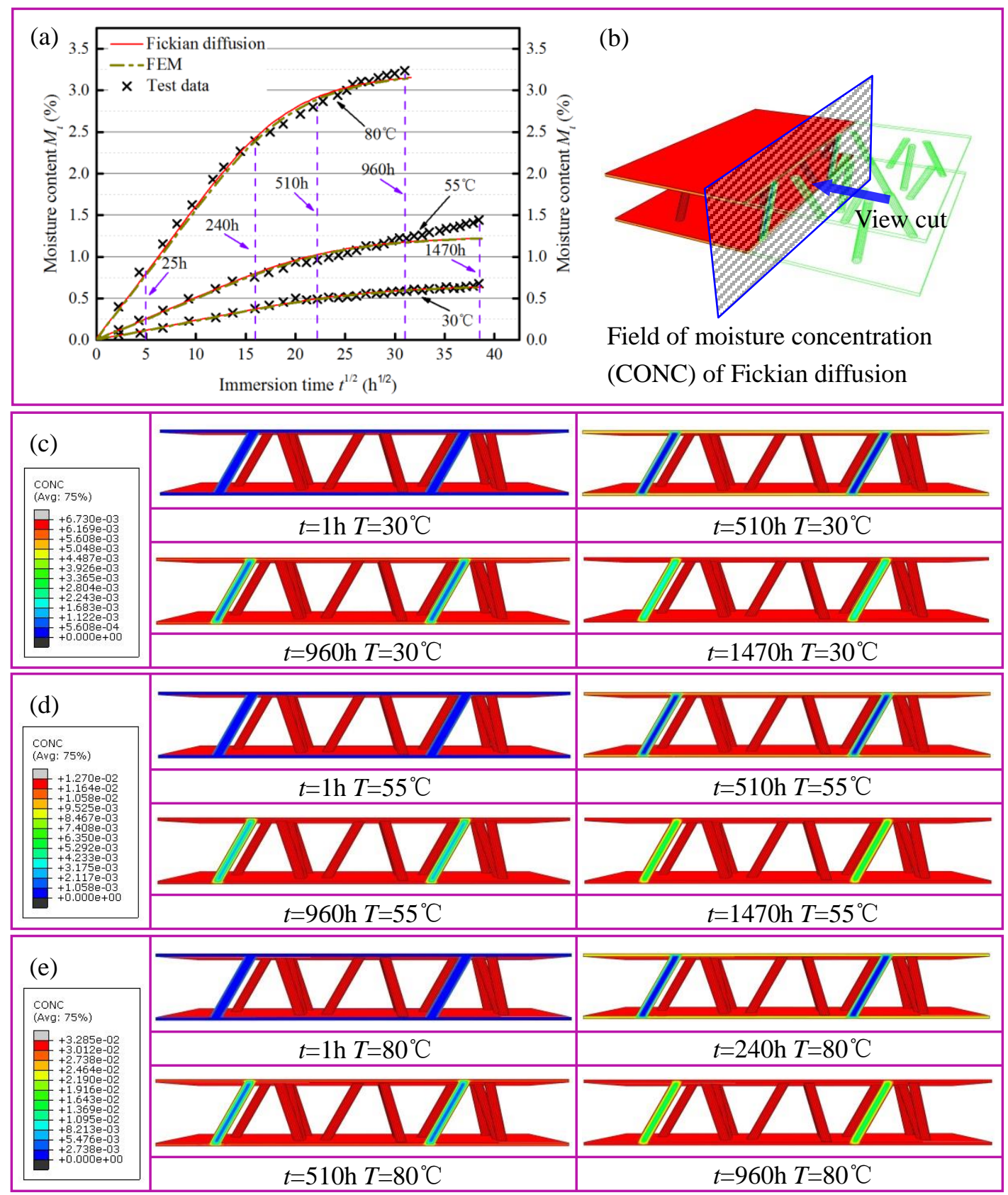

Fig. 13. (a) moisture diffusion curves of FEM and analytical model of Fickian diffusion; (b) diagrammatic view cut position of FEM results; (c) field of moisture concentration at $30^{\circ} \mathrm{C}$ of Fickian diffusion; (d) field of moisture concentration at $55^{\circ} \mathrm{C}$ of Fickian diffusion; (e) field of moisture concentration at $80^{\circ} \mathrm{C}$ of Fickian diffusion. 


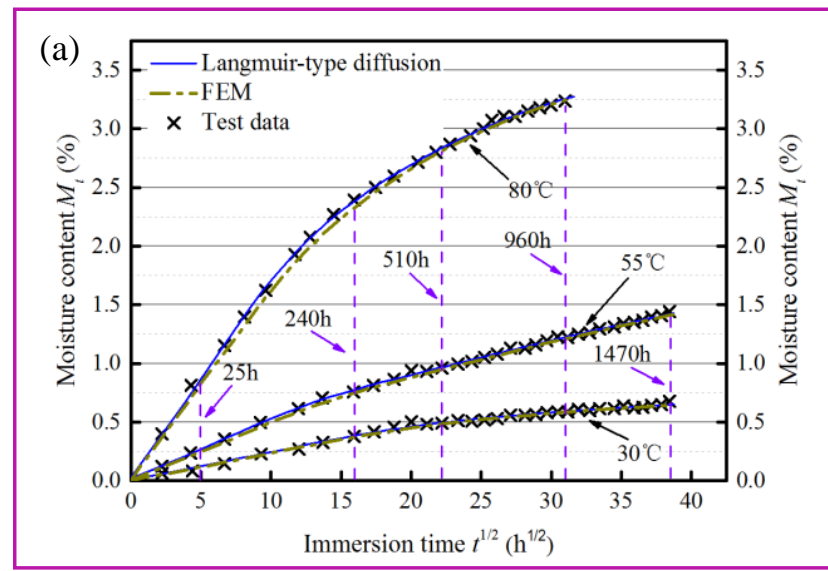

(b)
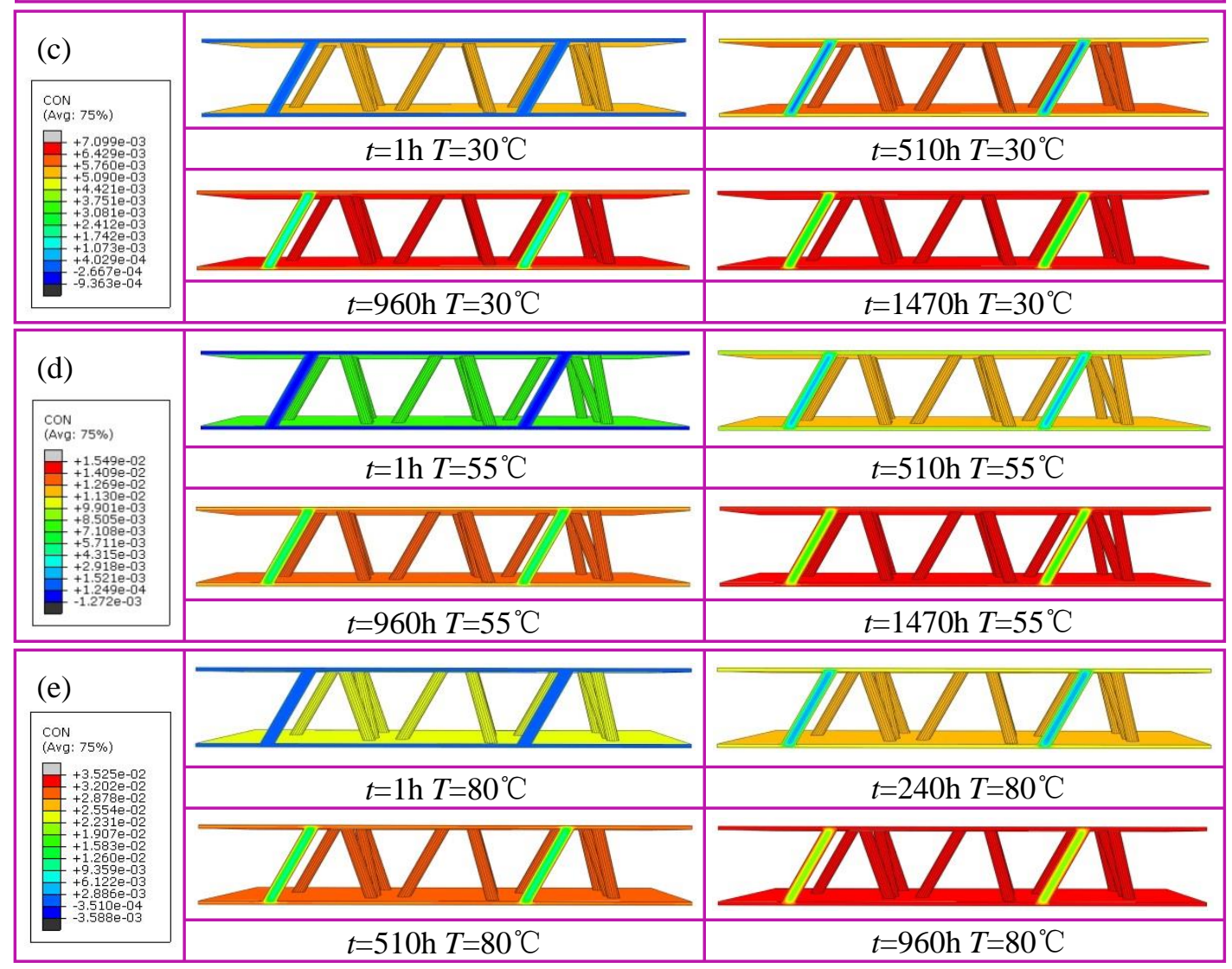

Fig. 14. (a) moisture diffusion curves of FEM and analytical model of Langmuir-type diffusion; (b) diagrammatic view cut position of FEM results; (c) field of moisture concentration at $30^{\circ} \mathrm{C}$ of Langmuir-type diffusion; (d) field of moisture concentration at $55^{\circ} \mathrm{C}$ of Langmuir-type diffusion; (e) field of moisture concentration at $80^{\circ} \mathrm{C}$ of Langmuir-type diffusion. 
(a)

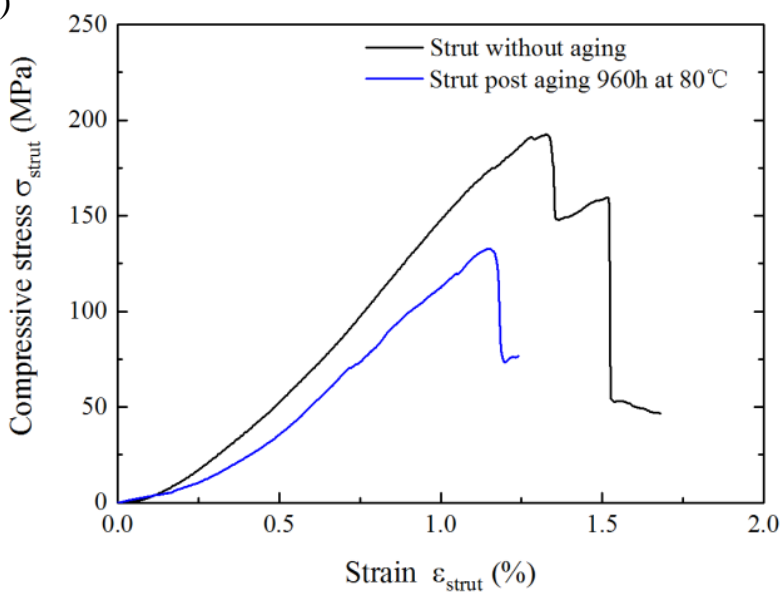

(b)

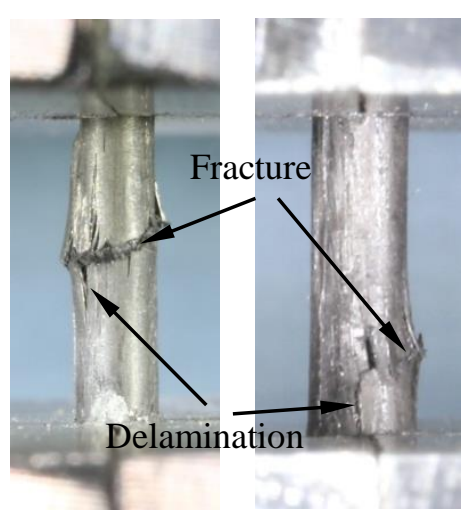

Fig. 15. (a) typical stress-strain curves of single struts under uniaxial compression; (b) typical failure mode of single struts in uniaxial compression testing.

(a)

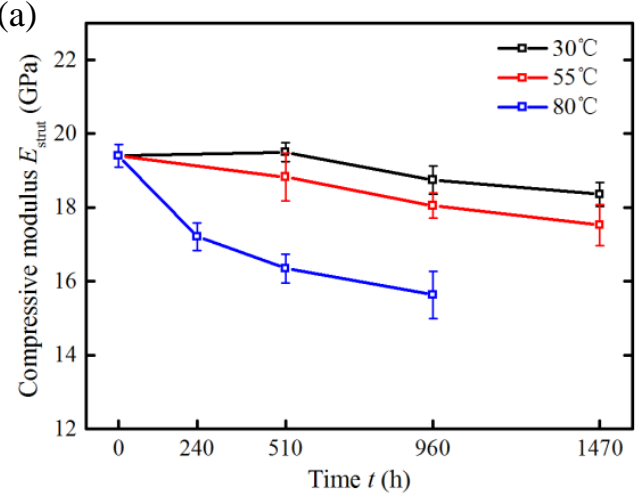

(b)

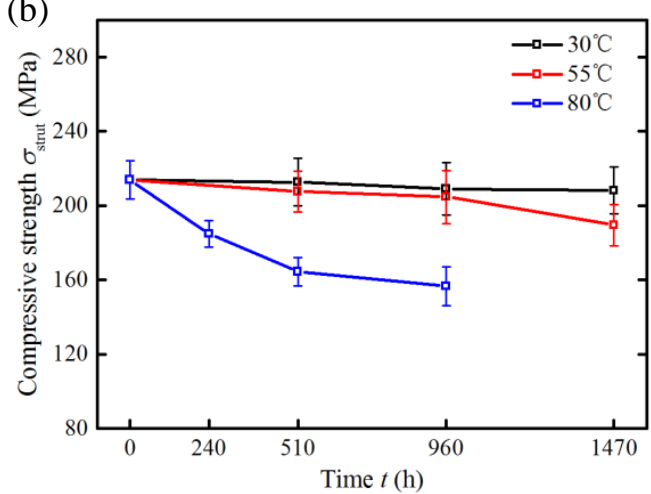

Fig. 16. (a) Variation of the (a) compressive modulus and (b) compressive strength with immersion time for a single strut at different temperature. 


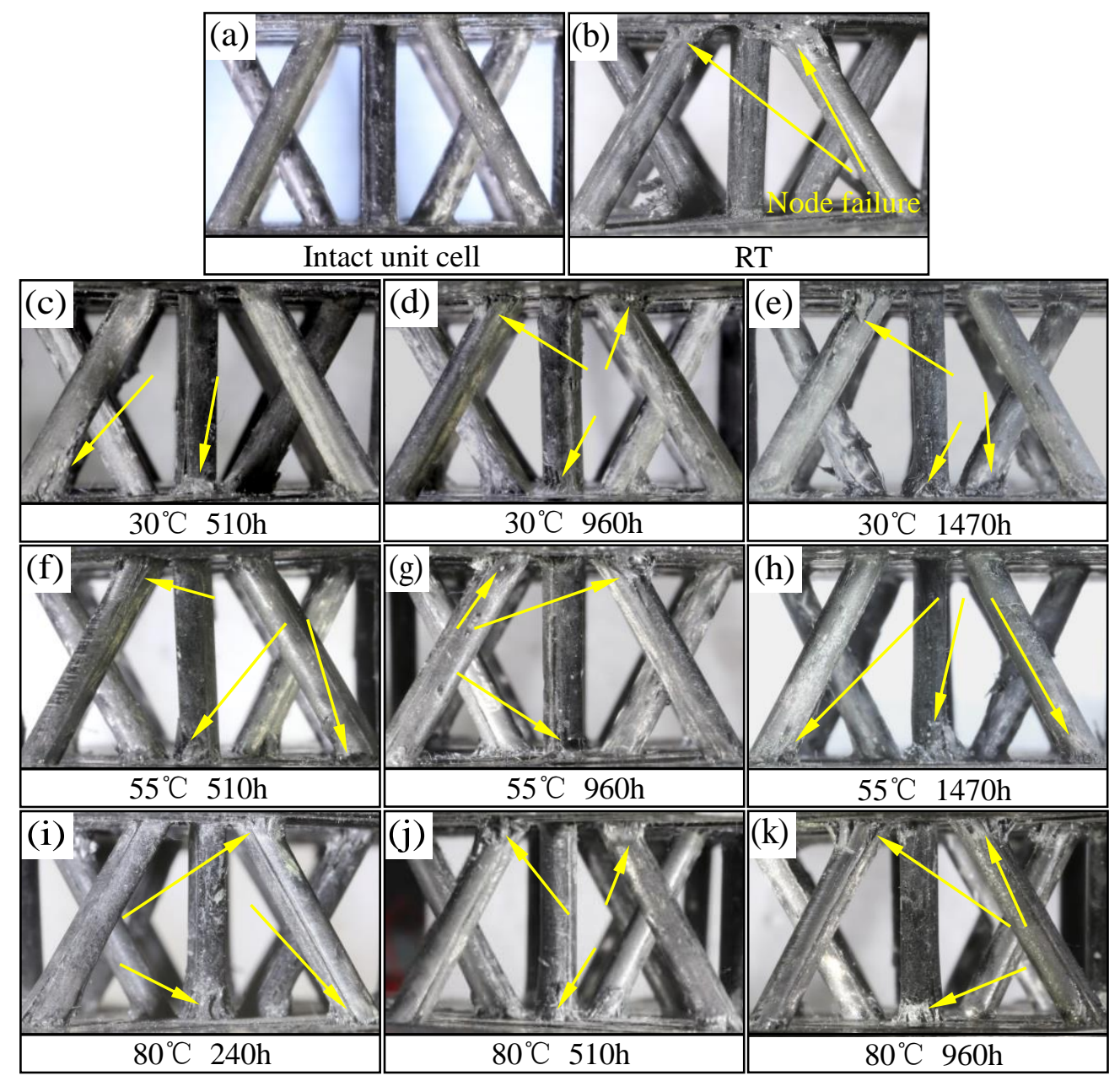

Fig. 17. Typical failure mode (node failure marked by yellow arrows) of the tetrahedral truss cores sandwich panel under out-of-plane compression of (a) intact truss cores; (b) compressive specimen without aging; (c-e) aged $510 \mathrm{~h}, 960 \mathrm{~h}$ and $1470 \mathrm{~h}$ in water at $30^{\circ} \mathrm{C}$; (f-h) aged $510 \mathrm{~h}, 960 \mathrm{~h}$ and $1470 \mathrm{~h}$ in water at $55^{\circ} \mathrm{C}$; (i-k) aged $240 \mathrm{~h}, 510 \mathrm{~h}$ and $960 \mathrm{~h}$ in water at $80^{\circ} \mathrm{C}$. 
(a)

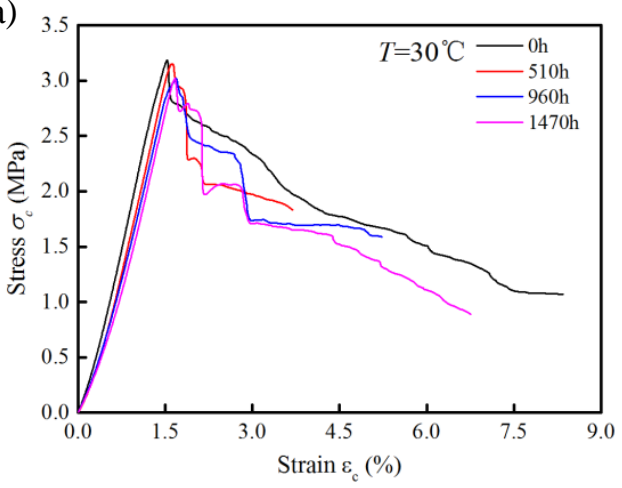

(c)

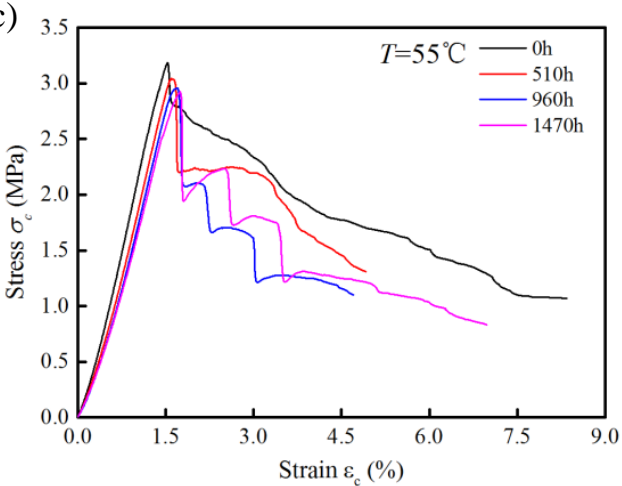

(e)

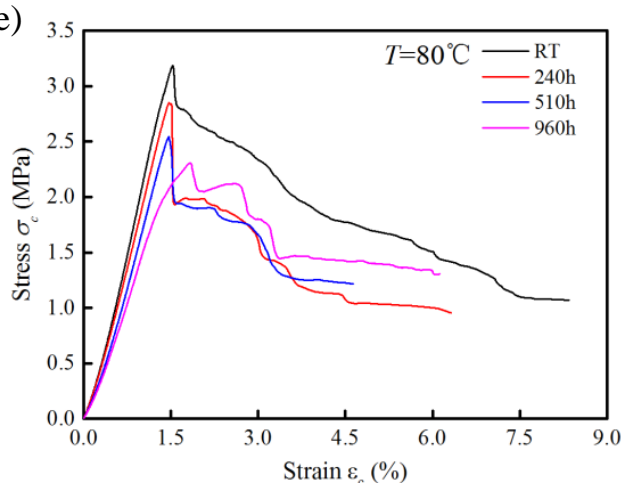

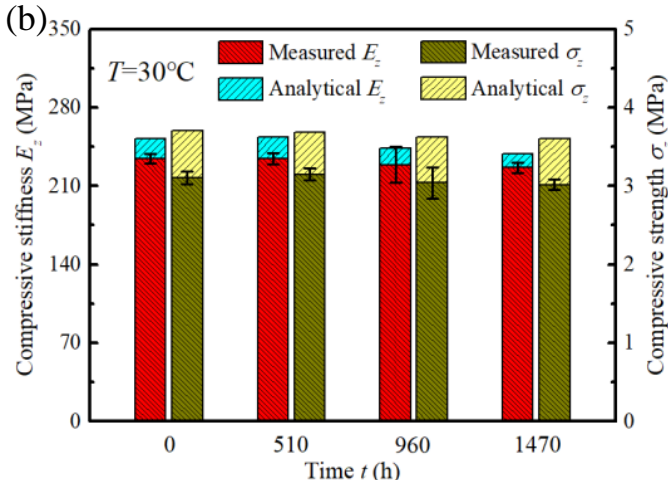
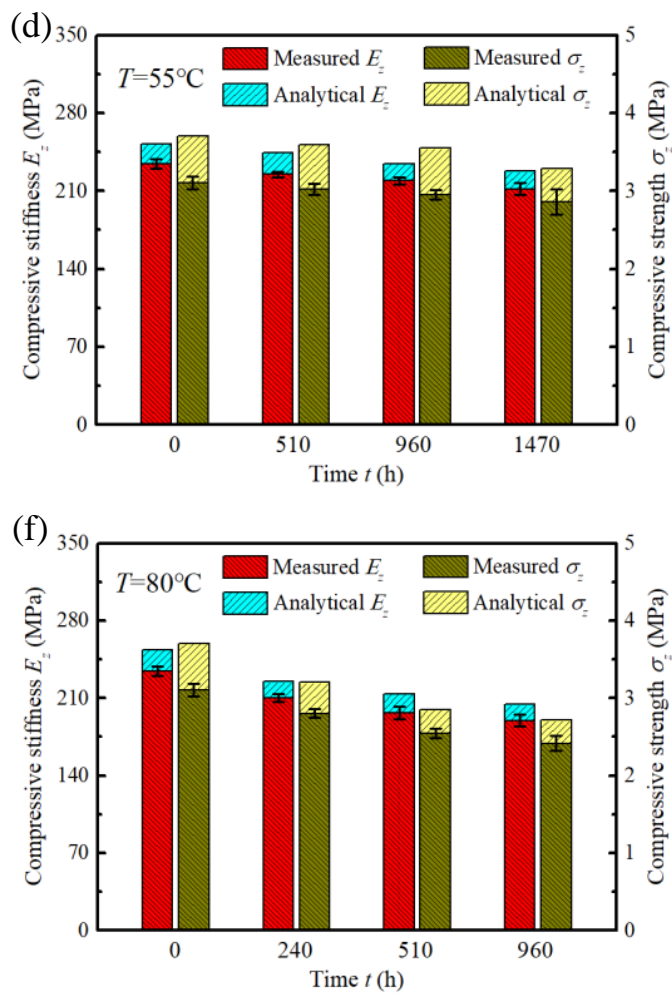

Fig. 18. (a) stress-strain curves of composite sandwich panel at $30^{\circ} \mathrm{C}$; (b) compressive stiffness and strength of composite sandwich panel over time at $30^{\circ} \mathrm{C}$. (c) Stress-strain curves of composite sandwich panel at $55^{\circ} \mathrm{C}$; (d) compressive stiffness and strength of composite sandwich panel over time at $55^{\circ} \mathrm{C}$. (e) Stress-strain curves of composite sandwich panel at $80^{\circ} \mathrm{C}$; (f) compressive stiffness and strength of composite sandwich panel over time at $80^{\circ} \mathrm{C}$. 

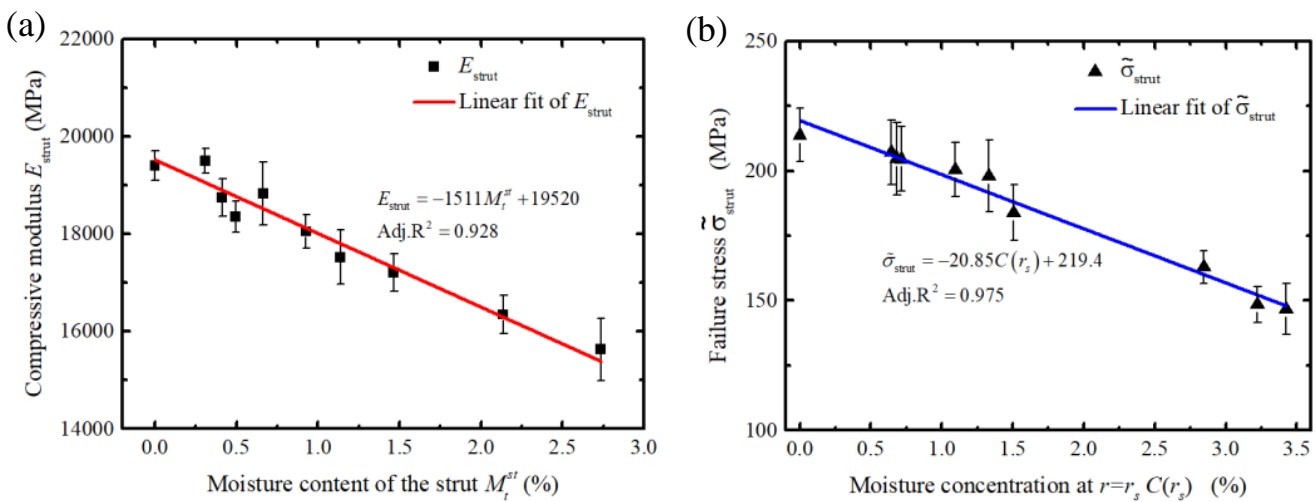

Fig. 19. (a) Linear fit of compressive modulus $E_{\text {strut }}$ and moisture content of the strut $M_{t}^{s t}$; (b) Linear fit of failure stress $\tilde{\sigma}_{\text {strut }}$ and moisture concentration $C\left(r_{s}\right)$.

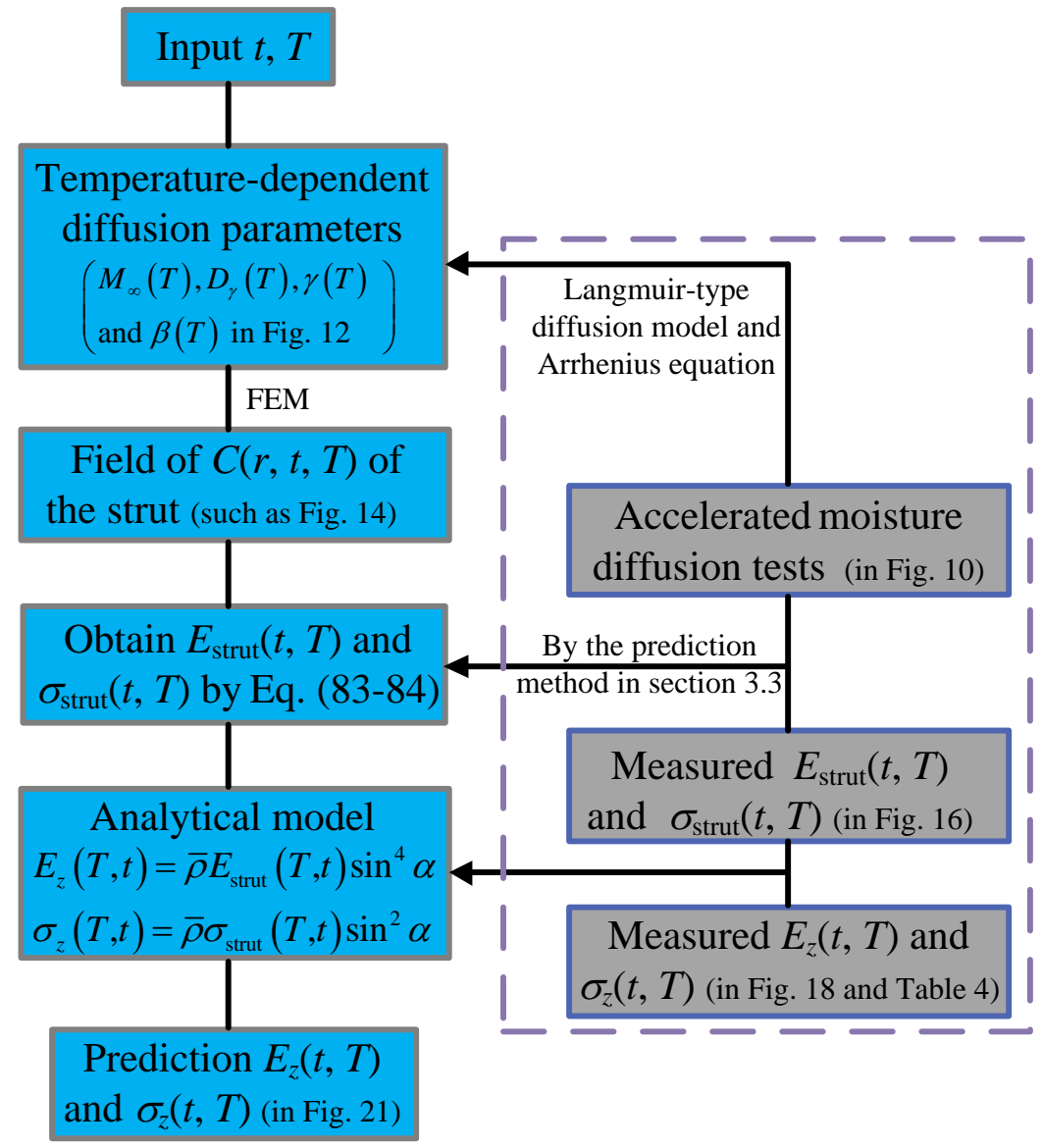

Fig. 20. Flowchart of the prediction method for compressive mechanical properties of the sandwich panel. 
(a)

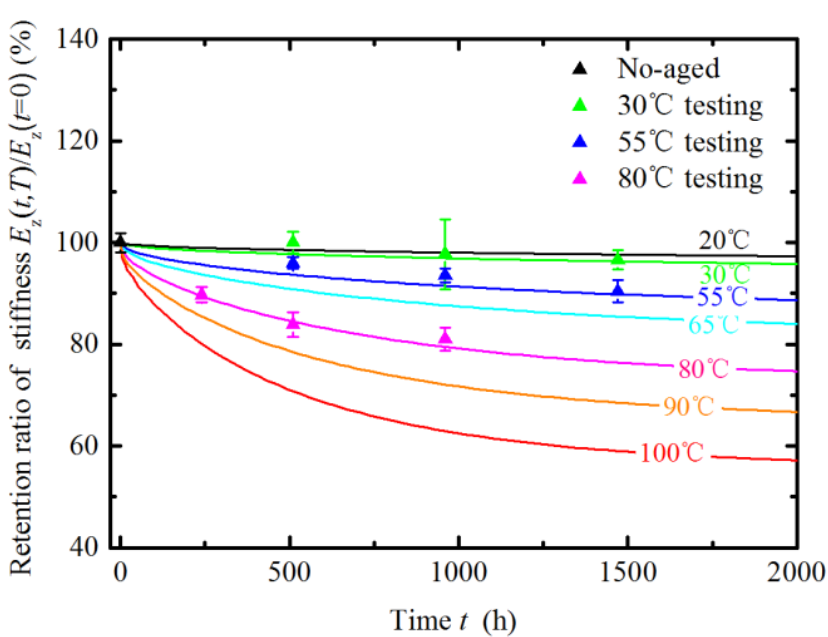

(b)

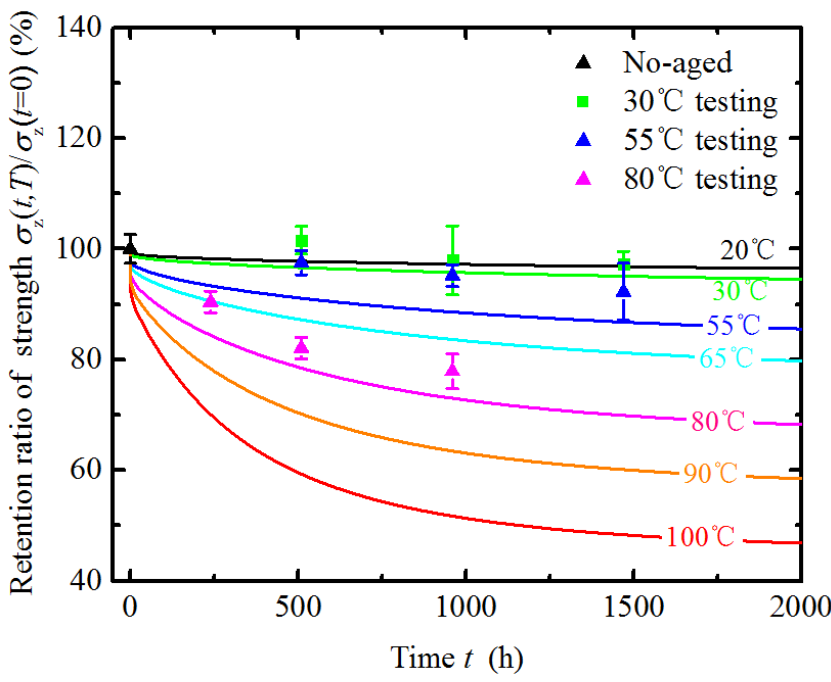

Fig. 21. (a) prediction retention ratio of compressive stiffness $E_{z}(t, T)$ of the sandwich panel at different time $t$ and temperature $T$; (b) Prediction retention ratio of compressive strength $\sigma_{z}(t, T)$ of the sandwich panel at different time $t$ and temperature $T$. 
Table 1: Relative density of sandwich panel (Eq. 2).

\begin{tabular}{|c|c|c|c|}
\hline Specimen & \multicolumn{2}{|c|}{ Relative density of specimens } & $\bar{\rho}_{r} \quad(\%)$ \\
\hline Temperature $T\left({ }^{\circ} \mathrm{C}\right)$ & 30 & 55 & 80 \\
\hline $\mathrm{S}-1-T$ & 1.52 & 1.52 & 1.63 \\
\hline $\mathrm{S}-2-T$ & 1.53 & 1.51 & 1.65 \\
\hline $\mathrm{S}-3-T$ & 1.59 & 1.57 & 1.67 \\
\hline
\end{tabular}

Table 2: Diffusion parameters at different temperatures for Fickian diffusion model.

\begin{tabular}{ccccc}
\hline Temperature $T\left({ }^{\circ} \mathrm{C}\right)$ & Specimen & $D\left(10^{-3} \mathrm{~mm}^{2} / \mathrm{h}\right)$ & $M_{\infty}(\%)$ & Residual error \\
\hline \multirow{3}{*}{30} & S-1-30 & 0.195 & 0.673 & 0.008 \\
& S-2-30 & 0.235 & 0.595 & 0.011 \\
& S-3-30 & 0.170 & 0.797 & 0.015 \\
\multirow{3}{*}{55} & S-1-55 & 0.260 & 1.270 & 0.240 \\
& S-2-55 & 0.259 & 1.235 & 0.215 \\
& S-3-55 & 0.234 & 1.247 & 0.229 \\
& S-1-80 & 0.391 & 3.282 & 0.114 \\
& S-2-80 & 0.349 & 3.349 & 0.038 \\
& S-3-80 & 0.382 & 3.309 & 0.134 \\
\hline
\end{tabular}


Table 3: Diffusion parameters at different temperatures for Langmuir-type diffusion model.

\begin{tabular}{ccccccc}
\hline $\begin{array}{c}\text { Temperature } T \\
\left({ }^{\circ} \mathrm{C}\right)\end{array}$ & Specimen & $\begin{array}{c}D_{\gamma} \\
\left(10^{-3} \mathrm{~mm}^{2} / \mathrm{h}\right)\end{array}$ & $\begin{array}{c}M_{\infty} \\
(\%)\end{array}$ & $\begin{array}{c}\gamma \\
\left(10^{-3} \mathrm{~h}^{-1}\right)\end{array}$ & $\begin{array}{c}\beta \\
\left(10^{-3} \mathrm{~h}^{-1}\right)\end{array}$ & $\begin{array}{c}\text { Residual } \\
\text { error }\end{array}$ \\
\hline \multirow{3}{*}{30} & $\mathrm{~S}-1-30$ & 0.304 & 0.840 & 0.280 & 0.535 & 0.005 \\
& $\mathrm{~S}-2-30$ & 0.351 & 0.729 & 0.236 & 0.527 & 0.008 \\
& $\mathrm{~S}-3-30$ & 0.205 & 0.860 & 0.120 & 0.654 & 0.014 \\
55 & $\mathrm{~S}-1-55$ & 0.715 & 1.872 & 1.1757 & 0.822 & 0.003 \\
& $\mathrm{~S}-2-55$ & 0.832 & 1.817 & 1.2477 & 0.817 & 0.004 \\
& $\mathrm{~S}-3-55$ & 0.856 & 1.877 & 1.4243 & 0.800 & 0.013 \\
& $\mathrm{~S}-1-80$ & 0.904 & 3.565 & 1.3344 & 2.350 & 0.010 \\
& $\mathrm{~S}-2-80$ & 0.849 & 3.386 & 2.5354 & 4.410 & 0.011 \\
& $\mathrm{~S}-3-80$ & 1.022 & 3.530 & 1.6360 & 2.620 & 0.016 \\
\hline
\end{tabular}

Table 4: Out-of-plane compression strength and stiffness of the sandwich panel.

\begin{tabular}{cccc}
\hline Temperature $\left({ }^{\circ} \mathrm{C}\right)$ & $\begin{array}{c}\text { Immersion } \\
\text { time }(\mathrm{h})\end{array}$ & $\begin{array}{c}\text { Compressive strength } \\
(\mathrm{MPa})\end{array}$ & $\begin{array}{c}\text { Compressive stiffness } \\
(\mathrm{MPa})\end{array}$ \\
\hline \multirow{3}{*}{30} & 510 & $3.15 \pm 0.08$ & $234.3 \pm 5.1$ \\
& 960 & $3.04 \pm 0.19$ & $229.0 \pm 16.1$ \\
& 1470 & $3.02 \pm 0.07$ & $226.2 \pm 4.5$ \\
55 & 510 & $3.02 \pm 0.07$ & $224.8 \pm 2.7$ \\
& 960 & $2.95 \pm 0.06$ & $219.1 \pm 3.1$ \\
& 1470 & $2.86 \pm 0.16$ & $211.8 \pm 5.3$ \\
80 & 240 & $2.80 \pm 0.06$ & $210.2 \pm 3.5$ \\
& 510 & $2.55 \pm 0.06$ & $196.4 \pm 5.7$ \\
& 960 & $2.41 \pm 0.10$ & $189.8 \pm 5.4$ \\
\hline
\end{tabular}

